\title{
A GENERAL CONDITION FOR LIFTING THEOREMS
}

\author{
E. ARTHUR ROBINSON, JR.
}

\begin{abstract}
We define a general condition, called stability on extensions $T$ of measure preserving transformations $S$. Stability is defined in terms of relative unique ergodicity, and as a joining property. Ergodic compact group extensions are stable, and moreover stable extensions satisfy lifting theorems similar to those satisfied by group extensions. In general, stable extensions have relative entropy zero. In the class of continuous flow extensions over strictly ergodic homeomorphisms, stable extensions are generic.
\end{abstract}

\section{INTRODUCTION}

In many cases, ergodic properties pass from extensions to factors. However, the converse is rarely true. Ergodic properties usually do not lift form factors to extensions unless the extension satisfies some additional conditions. It is these additional conditions which we wish to study in this paper. We will refer to such conditions quite generally as lifting conditions. Of course many very specific lifting conditions exist, but they usually fail to be of much interest. The point is to find lifting conditions which are as general as possible, both in terms of how prevalent they are and how many ergodic properties they lift. The best known example of the kind of general lifting condition we will be interested in is for $T$ to be a weakly mixing compact group extension of $S$. Such extensions satisfy many "lifting theorems", i.e., a great many ergodic properties, including mild mixing [29], multiple mixing [32], the $K$-property [26], and the Bernoulli property [31], automatically lift from $S$ to $T$. Moreover, at least within the class of all group extensions, those which are weakly mixing are generic [20, 28].

In this paper we study a general condition for extensions $T$ of $S$ called stability which generalizes the condition of being a group extension. In particular, every ergodic group extension is a stable extension, and like group extensions, stable extensions satisfy many lifting theorems.

In Part I of the paper we define stability in terms of a more general property of extensions: relative unique ergodicity. Group extensions are well-known to be relatively uniquely ergodic, and it turns out that many of the proofs of lifting theorems in the group extension case (at least implicitly) involve this fact. However, even though a general definition of relative unique ergodicity is easily

Received by the editors January 1, 1990.

1980 Mathematics Subject Classification (1985 Revision). Primary 28D05; Secondary 28D20.

Key words and phrases. Ergodic theory.

Partially supported by NSF Grant DMS 8805340. 
formulated, it is quickly seen that in itself it is insufficient to imply any lifting theorems. Our definition of stability strengthens the definition of relative unique ergodicity enough to overcome this insufficiency.

After defining stability we study the basic properties of stable extensions. An important step is to characterize stability in terms of the theory of joinings. This allows us to show that stability is essentially an isomorphism invariant property (unlike relative unique ergodicity). The theory of joinings also provides an important link to lifting theorems. We show that many of the lifting theorems already known for group extensions generalize to stable extensions, and we obtain some new lifting theorems as well. In particular we obtain lifting theorems for the $K$-property, mild mixing, strong mixing, and partial mixing. The question of whether or not the Bernoulli property lifts to stable extensions remains open.

Among the ergodic properties which lift to stable extensions is a class of properties which behave like the $K$-property in the sense that their 'complements' satisfy an analogue of the Pinsker Lemma. We call these properties natural properties. Our study of natural properties leads us to consider Glasner's theory of quasifactors [16]. In addition to the $K$-property, natural properties include weak mixing, mild mixing and, somewhat surprisingly, entropy zero.

In Part II of the paper we ask the general question, what extensions are stable? It is easy and natural to generalize the proof that group extensions are stable to the case of arbitrary distal extensions. We also study the special case of compact affine extensions. We show that a compact affine extension is distal if and only if it has relative entropy zero, or equivalently, the automorphism involved has topological entropy zero. We then prove that in general stable extensions have relative entropy zero. In particular, this raises the question of whether stable extensions are really the same as distal extensions, leading us to look for examples of stable extensions which are not distal.

In their paper $O n \mathscr{W}^{\perp}$ [18], Glasner and Weiss show that the generic "flow extension" $T$ over an irrational rotation $S$ is not distal and also satisfies a certain property which we call the $G W$-property. We show that the $G W$-property implies stability, giving a negative answer to our questions. However, this answer is not completely satisfying from the point of view of lifting theorems because the examples produced this way always have an irrational rotation factor. Our major result in Part II extends the result of [18] to the weakly mixing case. We show that the generic flow extension $T$ of a weakly mixing transformation $S$ is stable, assuming only that the flow involved is uniquely ergodic. If the flow is also weakly mixing, then in combination with [18], we get weakly mixing nondistal stable extensions.

As we noted above, our main goal in this paper is to examine the extent to which more general sorts of extensions satisfy the kind of lifting theorems which group extensions satisfy. A different approach to the same goal is pursued in Rudolph's paper [33] on lifting the $K$-property to certain $\mathbf{R}^{n}$ and $\mathbf{Z}^{n}$ action extensions. Rudolph's results are both more and less general than ours. In particular, he specifically uses the fact that the $K$-property and weak mixing are what we call natural properties. However, the exact relationship between Rudolph's results and ours remains unclear.

Because we prove a genericity theorem for stability, the results in this paper are also related to the many results stating that ergodic properties lift to various 
sorts of "generic" extensions. An early example of such a result says that ergodicity and weak mixing lift generically to compact abelian group extensions [20]. Later more general examples of results of this type appear in $[25,17$, and 28].

\section{PART I. THE PROPERTIES OF STABLE EXTENSIONS}

\section{Preliminaries}

2.1. Basic definitions. Our basic objects of study in this paper will be extensions of measure preserving dynamical systems. Although many of our results hold in greater generality (i.e., for actions of $\mathbf{Z}^{n}$ and $\mathbf{R}^{n}$ ), we will regard a measure preserving dynamical system as a quadruple $(X, \mathscr{B}, \mu, T)$ where:

(i) $(X, \mathscr{B})$ is a standard Borel space, (cf. [23]), where $\mathscr{B}$ denotes the Borel $\sigma$ algebra on $X$.

(ii) $\mu$ is a probability measure on $(X, \mathscr{B})$.

A triple $(X, \mathscr{B}, \mu)$ consisting of a standard Borel space and a probability measure is called a Lebesgue probability space. ${ }^{1}$ When no confusion can arise we will sometimes denote $(X, \mathscr{B})$ or $(X, \mathscr{B}, \mu)$ by $X$. The set of all probability measures on $(X, \mathscr{B})$ will be denoted by $X^{*}$.

(iii) $T$ is a Borel automorphism of $(X, \mathscr{B})$ which preserves the measure $\mu$.

This means $\mu\left(T^{-1} E\right)=\mu(E)$ for all $E \in B$. Equivalently, $\mu$ is $T$-invariant. The set of all $T$-invariant measures $\mu$ will be denoted by $X_{T}^{*}$. Sometimes for simplicity we denote $(X, \mathscr{B}, \mu, T)$ by $T$, or refer to $T$ as a measure preserving transformation. A set $E \in \mathscr{B}$ is $T$-invariant if $T^{-1} E=E$. Two measure preserving transformations $(X, \mathscr{B}, \mu, T)$ and $(Y, \mathscr{A}, \nu, S)$ are isomorphic if there exist invariant subsets $X_{0} \subseteq X, Y_{0} \subseteq Y$, with $\mu\left(X_{0}\right)=\nu\left(Y_{0}\right)=1$, and a Borel mapping $\sigma: X_{0} \rightarrow Y_{0}$ such that $S \sigma=\sigma T$. We say $\sigma$ is an isomorphism from $T$ to $S$. Two measure preserving transformations $T$ and $T^{\prime}$ on the same space $(X, \mathscr{B}, \mu)$ are equivalent if they are isomorphic via $\sigma=\mathrm{Id}$, where Id denotes the identity map. If $X_{T}^{*}=\{\mu\}$ then $T$ is called uniquely ergodic. Clearly if $T$ is uniquely ergodic then it is ergodic. Unique ergodicity is generally not an invariant of equivalence or isomorphism.

Suppose $(X, \mathscr{B}, \mu)$ and $(Y, \mathscr{A}, \nu)$ are Lebesgue spaces and $\pi:(X, \mathscr{B}) \rightarrow$ $(Y, \mathscr{A})$ is a Borel mapping, i.e. $\pi^{-1} \mathscr{A} \subseteq \mathscr{B}$. We define $\pi^{*}: X^{*} \rightarrow Y^{*}$ by

$$
\left(\pi^{*} \mu\right)(E)=\mu\left(\varphi^{-1} E\right) \text {. }
$$

We say that $\pi$ is a factor map if $\left(\pi^{*}\right)^{-1}\{\nu\} \neq \varnothing$. We say $\pi$ is measure preserving if $\nu=\pi^{*} \mu$. Notice that every measure preserving map is a factor map. In this paper we consider only factor maps which are measure preserving. For a measure preserving factor map $\pi$ we let

$$
X^{*} \mid Y=\left\{\zeta \in X^{*}: \pi^{*} \zeta=\nu\right\}
$$

noting that $\mu \in X^{*} \mid Y$. The measures $\zeta \in X^{*} \mid Y$ will be called a lifts of $\nu$.

\footnotetext{
${ }^{1}$ Our definition of a Lebesgue space, which is equivalent to (but not identical to) the usual definition, follows [42]. The usual definition is the completion $(\widehat{X}, \widehat{B}, \hat{\mu})$ of $(X, \mathscr{B})$ with respect to the measure $\mu$.
} 
The most basic example of a factor map between Lebesgue spaces is the projection map to a coordinate in a product space. Let $(Y, \mathscr{A}, \nu)$ and $(Z, \mathscr{C}, \omega)$ be Lebesgue spaces and let $(X, \mathscr{B}, \mu)=(Y, \mathscr{A}, \nu) \times(Z, \mathscr{C}, \omega)$ be the product space. Let $\pi(x)=\pi(y, z)=y$ be the projection map to the first coordinate. Clearly $\pi$ is a measure preserving factor map. In this situation we have the following well-known disintegration theorem.

Lemma 2.1. For any $\theta \in(Y \times Z)^{*} \mid Y$ there exists a mapping $y \mapsto \theta_{y}: Y \rightarrow Z^{*}$, unique $\nu$ a.e., such that for any bounded measurable function $f$

$$
\int_{Y \times Z} f(y, z) d \theta=\int_{Y} \int_{Z} f(y, z) d \theta_{y}(z) d \nu(y) .
$$

Given two measure preserving dynamical systems $(X, \mathscr{B}, \mu, T)$ and $(Y$, $\mathscr{A}, \nu, S)$, suppose $\pi:(X, \mathscr{B}, \mu, T) \rightarrow(Y, \mathscr{A}, \nu)$ is a measure preserving factor map satisfying $S \pi=\pi T$. We say that $(Y, \mathscr{A}, \nu, S)$ is a factor of $(X, \mathscr{B}, \mu, T)$, and $(X, \mathscr{B}, \mu, T)$ is an extension of $(Y, \mathscr{A}, \nu, S)$. We denote this by $T \stackrel{\pi}{\rightarrow} S$ or $T \rightarrow S$. For a given extension $T \stackrel{\pi}{\rightarrow} S$, let us define

$$
X_{T}^{*}\left|Y=X^{*}\right| Y \cap X_{T}^{*} \text {. }
$$

We call the measures $\mu \in X_{T}^{*} \mid Y$ relative invariant measures. ${ }^{2}$

Now suppose $T \stackrel{\pi}{\rightarrow} S$ and $T^{\prime} \stackrel{\pi^{\prime}}{\rightarrow} S^{\prime}$ are extensions. We say that the two extensions are isomorphic if there exists an isomorphism $\sigma$ from $T$ to $T^{\prime}$ and an isomorphism $\bar{\sigma}$ from $S$ to $S^{\prime}$ such that $\bar{\sigma} \pi(x)=\pi \sigma(x)$, for all $x$ in an invariant set $X_{1}=\pi^{-1}\left(Y_{0}\right)$, where $Y_{0}$ is $S$ the invariant set of full measure from the definition of the isomorphism $\bar{\sigma}$.

Note. This is more than just saying that $T$ is isomorphic to $T^{\prime}$ and $S$ is isomorphic to $S^{\prime}$.

2.2. Some topological considerations. Every standard Borel space can be given a compact metric topology compatible with its Borel structure (cf., [23]). Although this topology is highly nonunique, there is a natural unique standard Borel structure $\mathscr{B}^{*}$ on $X^{*}$-it turns out to be the Borel structure induced by the weak-* topology on $X^{*}$ corresponding to any compatible compact metric topology on $(X, \mathscr{B}),($ cf., [37]). For a Borel automorphism $T$ on a standard Borel space $(X, \mathscr{B})$, we define $T^{*}:\left(X^{*}, \mathscr{B}^{*}\right) \rightarrow\left(X^{*}, \mathscr{B}^{*}\right)$ by

$$
T^{*} \mu(E)=\mu\left(T^{-1} E\right) \text {. }
$$

Then $T^{*}$ turns out to be a Borel automorphism of $\left(X^{*}, \mathscr{B}^{*}\right)$.

Usually more topological structure will be necessary for our purposes. Suppose $(X, \mathscr{B})$ has been given a compatible compact metric topology, and that $T$ is a measure preserving homeomorphism of $X$. We say that $(X, \mathscr{B}, \mu, T)$ is a topological dynamical system. Clearly in this case $T^{*}$ is a homeomorphism of $X^{*}$ in the weak-* topology. We say $T$ is minimal if every point $x \in X$ has a dense orbit, and $T$ is strictly ergodic if it is minimal and uniquely ergodic. In many situations we may assume strict ergodicity without loss of generality: the Jewitt-Kreiger Theorem [22] says that any ergodic transformation is isomorphic to a strictly ergodic homeomorphism of a compact metric space. The following more general result is due to Weiss [41].

\footnotetext{
${ }^{2} \mathrm{~A}$ slightly different notion of a relative invariant measure is studied in topological dynamics (cf., [15]).
} 
Theorem 2.2. Every extension $T \stackrel{\pi}{\rightarrow} S$, with $T$ ergodic, is isomorphic to an extension $T \stackrel{\pi}{\rightarrow} S$ where $T^{\prime}$ and $S^{\prime}$ are strictly ergodic topological dynamical systems, and the factor map $\pi^{\prime}$ is continuous.

Thus we may assume that an arbitrary extension $T \stackrel{\pi}{\rightarrow} S$ has a topological model which is valid up to isomorphism. Fixing such a model, we will give $X^{*}$ and $Y^{*}$ the corresponding weak-* topologies. This leads to the following lemma.

Lemma 2.3. If $T \rightarrow S$ is an extension with $T$ ergodic, then there exists a choice of metrics $d_{X^{*}}$ on $X^{*}$ and $d_{Y^{*}}$ on $Y^{*}$ such that the maps $T^{*}, S^{*}$, and $\pi^{*}: X^{*} \rightarrow Y^{*}$ are continuous and affine.

Corollary 2.4. The sets $X^{*} \mid Y, X_{T}^{*}$, and $X_{T}^{*} \mid Y$ are closed and convex.

2.3. Skew-product extensions and cohomology. Given a measure preserving dynamical system $(Y, \mathscr{A}, \nu, S)$ and a Lebesgue probability space $(Z, \mathscr{C}, \omega)$, let $(X, \mathscr{B}, \mu)=(Y, \mathscr{A}, \nu) \times(Z, \mathscr{C}, \omega)$. For each $y \in Y$ let $R_{y}$ be a measure preserving Borel automorphism of $(Z, \mathscr{C}, \omega)$, where the dependence on $y \in Y$ is measurable in the sense that

$$
T(y, z)=\left(S y, R_{y} z\right),
$$

is a Borel automorphism. Then (5) defines a measure preserving dynamical system $(X, \mathscr{B}, \mu, T)$ which is called a skew-product extension. The following standard result is usually attributed to Rohlin:

Lemma 2.5. Any ergodic extension $T^{\prime} \rightarrow S^{\prime}$ is isomorphic to a skew-product extension $T \rightarrow S$, of the form (5).

In spite of Lemma 2.5, the general skew-product extension (5) is seldom of any practical use. More typically we have the following situation. Let $G$ be a locally compact group, and let $\left(Z, \mathscr{C}, \omega, h^{g}\right), g \in G$, be an ergodic measure preserving action of $G$ on a Lebesgue space. Given a mapping $\varphi: Y \rightarrow G$ we define a skew product

$$
T(y, z)=\left(S y, h^{\varphi(y)} z\right)
$$

called an action extension. The map $\varphi$ is called the cocycle of the extension. A cocycle $\varphi$ is called a coboundary if there exists a measurable function $\psi$ : $Y \rightarrow G$ such that $\varphi(y)=\psi(S y) \psi^{-1}(y)$ and two cocycles $\varphi_{1}$ and $\varphi_{2}$ are called cohomologous if $\varphi_{1}(y)=\psi(S y) \varphi_{1}(y) \psi^{-1}(y)$ for some $\psi$ as above (these equations are only required to hold $\bmod \nu$ ). If $G$ is abelian we often switch to additive notation. In that case two cocycles are cohomologous if they differ by a coboundary. Some important special cases of action extensions are the following:

(i) If $G=\mathbf{Z}$, the group of integers, $\varphi(y)=1$ for all $y \in Y$, and $h^{1}=R$ then (6) defines the Cartesian product $T=S \times R$.

(ii) If $G$ is a compact metric group then it acts transitively on itself $Z=G$ by translation. This action preserves normalized Haar measure $\omega$. In this case $T$ is a compact group extension and has the form

$$
T(y, g)=(S y, \varphi(y) g) \text {. }
$$

(iii) If $G=\mathbf{R}$, the group of real numbers, then $h^{s}$ is a flow and the corresponding extension (6) is called a flow extension. 


\section{Stable extensions}

There are two equivalent ways to define stability: (1) via relative unique ergodicity, and (2) as a joining property. Since each has certain advantages we will discuss both of them. We begin with relative unique ergodicity.

3.1. Relative unique ergodicity. Suppose $(X, \mathscr{B}, \mu, T)$ and $(Y, \mathscr{A}, \nu, S)$ are measure preserving dynamical systems such that $T$ is an extension of $S$ corresponding to a factor map $\pi$, or in other words $T \stackrel{\pi}{\rightarrow} S$. Since we always assume a factor map $\pi$ is measure preserving, we have $\mu \in X_{T}^{*} \mid Y$. If $\mu$ is the only relative invariant measure, that is if

$$
X_{T}^{*} \mid Y=\{\mu\},
$$

then we say the extension is relatively uniquely ergodic (or RUE for short). The following examples illustrate this concept:

(i) The trivial extension $T \rightarrow T$ is RUE.

(ii) If $S$ denotes the trivial dynamical system (i.e., if $S$ is the identity on $Y=\{y\})$, then $T \rightarrow S$ is RUE if and only if $T$ is uniquely ergodic.

(iii) Let $S$ and $R$ be disjoint (cf., $\S 3.5$ ) measure preserving transformations. If $R$ is uniquely ergodic then $S \times R \rightarrow S$ is RUE.

(iv) The best known example of an RUE extension is given by the following theorem:

Theorem 3.1 (Furstenberg [11]). If $T \rightarrow S$ is an ergodic compact group extension (7), then $T \rightarrow S$ is RUE with $X_{T}^{*} \mid Y=\{\nu \times \omega\}$.

Proof. For any $\theta \in X^{*} \mid Y$, Proposition 2.1 implies

$$
\theta=\int_{Y} \theta_{y}(g) d \nu(y) \text {. }
$$

Letting $R^{g}\left(y, g^{\prime}\right)=\left(y, r_{g} g^{\prime}\right)=\left(y, g^{\prime} g\right)$ we have

$$
\left(R^{g}\right)^{*} \theta=\int_{Y} r_{g}^{*} \theta_{y} d \nu(y),
$$

so that by the uniqueness of Haar measure

$$
\begin{aligned}
\bar{\theta} & \stackrel{\text { def }}{=} \int_{G}\left(R^{g}\right)^{*} \theta d \omega(g)=\int_{Y} \int_{G} r_{g}^{*} \theta_{y} d \omega(g) d \nu(y) \\
& =\int_{Y} \omega d \nu(y)=\nu \times \omega .
\end{aligned}
$$

Thus $\bar{\theta}$ is ergodic. It follows that $\theta=\bar{\theta}$ by the extremality of ergodic measures.

The next example generalizes Example (i) above.

(v) If $T$ is uniquely ergodic then $T \rightarrow S$ is RUE for any factor $S$.

In particular, using the Jewett-Kreiger Theorem [22], it is possible to construct a strictly ergodic homeomorphism $T^{\prime}$ isomorphic to any ergodic transformation $T$. It follows that given any factor $S$ of $T$, we have $T^{\prime} \stackrel{\pi^{\prime}}{\rightarrow} S$ is a RUE extension isomorphic to $T \stackrel{\pi}{\rightarrow} S$, for $\pi^{\prime}=\pi \circ \sigma^{-1}$, where $\sigma$ is the isomorphism from $T$ to $T^{\prime}$. Thus we have proven the following: 
Proposition 3.2. Any extension $T \rightarrow S$ is isomorphic to an RUE extension.

Corollary 3.3. For any measure preserving system $(Y, \mathscr{A}, \nu, S)$ there exists a nontrivial extension $T \rightarrow S$ which is $R U E$.

Proposition 3.2 shows that RUE is not an isomorphism invariant. In particular, this implies that in itself, RUE is not sufficient to imply any lifting theorems.

Proposition 3.4. If $S$ is ergodic and $T \rightarrow S$ is RUE then $T$ is ergodic.

3.2. The first definition of stability. In this section we introduce the property we call stability together with a closely related property called self-stability. Both of these properties are based on RUE, but turn out to be considerably stronger.

Definition 3.5. Let $T \rightarrow S$ be an extension with $T$ ergodic. If $T \times R \rightarrow S \times R$ is RUE for all $R$ such that $T \times R$ is ergodic, then the extension $T \rightarrow S$ is said to be stable.

For a measure preserving system $T$ let $T^{(r)}$ denote the $r$ th Cartesian power of $T$.

Definition 3.6. Let $T \rightarrow S$ be an extension with $S$ weakly mixing. The extension $T \rightarrow S$ is said to be $r$-fold self-stable if $T^{(r)} \rightarrow S^{(r)}$ is RUE. The extension $T \rightarrow S$ is said to be self-stable if it is $r$-fold self-stable for all $r$.

\subsection{Basic properties of stable and self-stable extensions.}

\section{Lemma 3.7.}

(i) If $T \rightarrow S$ is stable then it is RUE.

(ii) If $T \rightarrow S$ is stable and $T \times R$ is ergodic, then $T \times R \rightarrow S \times R$ is stable.

(iii) If $T \rightarrow S$ is stable and $T$ is weakly mixing then it is self-stable.

(iv) If $T \rightarrow S$ is 2-fold self-stable and $S$ is weakly mixing then $T$ is weakly mixing.

(v) If $T \rightarrow S$ is $r$-fold self-stable, then it is $s$-fold self-stable for all $s \leq r$.

Proof. (i) Take $R$ trivial. (ii) This is clear. (iii) By induction we may assume $T^{(r-1)} \rightarrow S^{(s-1)}$ is stable. Then both of the extensions in

$$
T^{(r)}=T^{(r-1)} \times T \rightarrow T^{(r-1)} \times S \rightarrow S^{(r-1)} \times S=S^{(r)},
$$

are stable and therefore RUE by (i). (iv) Self-stability for $T \rightarrow S$ implies RUE for $T \times T \rightarrow S \times S$, which implies $T \times T$ is ergodic by Proposition 3.4. (v) If $T^{(r-1)} \rightarrow S^{(r-1)}$ is not RUE then there exist $\rho, \rho^{\prime} \in\left(X^{r-1}\right)^{*} \mid Y^{r-1}$ with $\rho \neq \rho^{\prime}$. It follows that $\rho \times \nu, \rho^{\prime} \times \nu \in\left(X^{r}\right)^{*} \mid Y^{r}$ and $\rho \times \nu \neq \rho^{\prime} \times \nu$, contradicting $r$-fold self stability.

\section{Two open questions.}

1. Does 2 fold self-stability imply self-stability?

2. Does self-stability imply stability?

\subsection{Examples and counterexamples.}

Corollary 3.8. An ergodic compact group extension $T \rightarrow S$ is stable. A weakly mixing compact group extension is self-stable.

Proof. If $T \rightarrow S$ is an ergodic compact group extension and $T \times R$ is ergodic, then $T \times R \rightarrow S \times R$ is an ergodic compact group extension, so it is RUE by Lemma 3.1. The second statement follows from (iii) of Lemma 3.7. 
Not every RUE extension is stable. Consider for example (iii) in $\S 3.1$, taking $R=Q \times Q$. As we note below in Lemma 3.10, a Cartesian square can never be uniquely ergodic. Thus let $\omega$ denote the product measure and let $\omega_{1}$ denote any other $Q \times Q$ invariant measure. Both $\nu \times \omega$ and $\nu \times \omega_{1}$ project to $\nu$, which implies $S \times R \rightarrow S$ is not stable.

3.5. Some basic facts from the theory of joinings. In preparation for our characterization of stability as a joining property, and subsequently the proofs of lifting theorems we collect some basic facts and definitions from the theory of joinings.

Suppose $T=S_{1} \times S_{2} \times \cdots \times S_{r}$ is a direct product of $r$ measure preserving transformations on the product Lebesgue space $X=Y_{1} \times \cdots \times Y_{r}$. We define

$$
\mathscr{J}\left(S_{1}, S_{2}, \ldots, S_{r}\right)=X_{T}^{*}\left|Y_{1} \cap \cdots \cap X_{T}^{*}\right| Y_{r} .
$$

The measures $\rho \in \mathcal{J}\left(S_{1}, S_{2}, \ldots, S_{r}\right)$ are called the joinings of $S_{1}, S_{2}, \ldots, S_{r}$. This idea is due to Furstenberg [10]. The set of joinings is never empty since the product measure $\mu_{1} \times \mu_{2} \times \cdots \times \mu_{r}$ is always a joining.

Two measure preserving transformations $S_{1}$ and $S_{2}$ are called disjoint [10], if $\mathscr{J}\left(S_{1}, S_{2}\right)=\left\{\mu_{1} \times \mu_{2}\right\}$, (i.e. the product is RUE with respect to both coordinate projections).

Theorem 3.9 (Furstenberg [10]). If $S_{1}$ and $S_{2}$ are disjoint then $S_{1}$ and $S_{2}$ have no common factors.

In particular, if $S_{1}$ and $S_{2}$ have a common factor $S$ then $\mathscr{J}\left(S_{1}, S_{2}\right)$ contains nonproduct joining called the relatively independent joining, (cf., [13]). Rudolph showed that the converse is false, [30].

Given a measure preserving transformation $S$, let $S_{1}=S_{2}=\cdots=S_{r}=S$. A joining $\rho \in \mathcal{J}\left(S_{1}, S_{2}, \ldots, S_{r}\right)$ is called an $r$-fold self-joining of $S$, (cf., [30]). We denote the set of all $r$-fold self-joinings of $S$ by $\mathscr{J}_{r}(S)$. In addition to the product self-joining, other $r$-fold self-joinings always exist. For example if $\mathbf{n}=\left(n^{1}, \ldots, n^{r}\right)$ we define the $\mathbf{n}$ th off diagonal self-joining to be the unique measure in $\mathscr{J}_{r}(S)$ satisfying

$$
\Delta_{n}\left(A_{1} \times A_{2} \times \cdots \times A_{r}\right)=\nu\left(S^{n^{1}} A_{1} \cap S^{n^{2}} A_{2} \cap \cdots \cap S^{n^{r}} A_{r}\right) .
$$

We denote the set of all such joinings by $J_{r}^{\Delta}(S)$. More generally, one can define a class of joinings called products of off diagonal joinings, cf. [30]. Essentially this amounts to a product of joinings of the form (12) on each of certain disjoint collections of coordinate indices. In the case $r=2$ we also write

$$
\Delta_{S^{n}}(A \times B)=\nu\left(S^{n} A \cap B\right) .
$$

The existence of joinings of the type (12) has the following easy interesting consequence.

Lemma 3.10. The Cartesian square $Q \times Q$ of a measure preserving transformation $Q$ is never uniquely ergodic.

Note. If $S_{1}$ and $S_{2}$ are uniquely ergodic and disjoint then $S_{1} \times S_{2}$ is uniquely ergodic.

3.6. Stability as a joining property. The following constitutes "second definition of stability" in terms of joinings. 
Definition 3.11. An extension $T \rightarrow S$ is weakly stable if for every measure preserving transformation $R$ and every ergodic joining $\rho$ of $T$ and $R$, if $\bar{\pi}^{*}(\rho)=\nu \times \omega$ then $\rho=\mu \times \omega$, where $\bar{\pi}: X \times Z \rightarrow Y \times Z$ is defined $\bar{\pi}(x, z)=(\pi(x), z)$.

One of the main advantages of weak stability is the following fact which sets it apart from RUE.

Proposition 3.12. Weak stability is an isomorphism invariant for extensions.

Proof. Let $T \rightarrow S$ and $T^{\prime} \rightarrow S^{\prime}$ be isomorphic extensions, with $\sigma$ an isomorphism from $T$ to $T^{\prime}$, and with $\bar{\sigma}$ the corresponding isomorphism from $S$ to $S^{\prime}$. Let $R$ be such that $T \times R$ is ergodic, and let $\sigma_{1}=\sigma \times$ Id and $\bar{\sigma}_{1}=\bar{\sigma} \times$ Id be the corresponding isomorphisms from $T \times R$ to $T^{\prime} \times R$ and $S \times R$ to $S^{\prime} \times R$. Now any two distinct joinings $\rho_{1}$ and $\rho_{2}$ of $T$ and $R$ are mapped by $\sigma_{1}^{*}$ to distinct joinings of $T^{\prime}$ and $R$. Furthermore, if $\rho$ is any joining of $T$ and $R$ which projects to the product joining $\nu \times \omega$ of $S$ and $R$, then $\sigma_{1}^{*} \rho$ projects to the product joining $\nu^{\prime} \times \omega$ of $S^{\prime}$ and $R$, since $\bar{\sigma}_{1}^{*}(\nu \times \omega)=\left(\bar{\sigma}^{*} \nu \times \omega\right)=\left(\nu^{\prime} \times \omega\right)$. It follows that $T \rightarrow S$ is not weakly stable if and only if $T^{\prime} \rightarrow S^{\prime}$ is not weakly stable.

The next proposition describes the relation between weak stability and stability.

Proposition 3.13. An extension $T \rightarrow S$ is stable if and only if it is weakly stable and RUE.

The proof depends on the following lemma which is of some independent interest.

Lemma 3.14. Let $T \stackrel{\pi}{\rightarrow} S$ be an RUE extension. Let $R$ be a measure preserving transformation and let $T \times R \stackrel{\pi}{\rightarrow} S \times R$. If $\rho$ is a $T \times R$ invariant measure such that $\bar{\pi}^{*}(\rho) \in \mathscr{J}(S, R)$, then $\rho \in \mathcal{J}(T, R)$.

In other words, RUE implies that joinings lift to joinings.

Proof. Let $\rho$ be as above and let $\rho^{\prime}=\bar{\pi}^{*}(\rho)$. Let $\bar{\pi}: X \times Z \rightarrow Y \times Z, \pi_{1}$ : $Y \times Z \rightarrow Y, \pi_{2}: Y \times Z \rightarrow Z, \pi_{X}: X \times Z \rightarrow X$, and $\pi_{Z}: X \times Z \rightarrow X$ all be the obvious projection maps. Now $\pi_{Z}=\pi_{1} \circ \bar{\pi}$, so that $\pi_{1}^{*}\left(\bar{\pi}^{*}(\rho)\right)=\pi_{1}^{*}\left(\rho^{\prime}\right)=\omega$, since $\rho^{\prime} \in \mathscr{J}(S, R)$. Thus we have $\pi_{Z}^{*}(\rho)=\omega$. Now $\pi \circ \pi_{X}=\pi_{Y} \circ \bar{\pi}$, so that $\pi^{*} \pi_{X}^{*}(\rho)=\pi_{Y}^{*} \bar{\pi}^{*}(\rho)=\pi_{Y}^{*}\left(\rho^{\prime}\right)=\nu$, again since $\rho^{\prime} \in \mathscr{J}(S, R)$. Thus, if $\pi_{X}^{*}(\rho)=\tilde{\rho}$, then $\pi^{*}(\tilde{\rho})=\nu$. It follows by RUE for $T \stackrel{\pi}{\rightarrow} S$ that $\rho^{\prime}=\mu$. Thus $\pi_{X}^{*}(\rho)=\mu$. Thus $\rho \in \mathscr{J}(T, R)$.

Proof of Proposition 3.13. Suppose $T \stackrel{\pi}{\rightarrow} S$ is stable. By (i) of Lemma 3.7, $T \stackrel{\pi}{\rightarrow} S$ is RUE. Furthermore, if $T \times R$ is ergodic then by weak stability, $\nu \times \omega$ lifts uniquely to $\mu \times \omega$.

Conversely, suppose $T \stackrel{\pi}{\rightarrow} S$ is weakly stable and RUE. Let $\rho \in$ $(X \times Z)^{*} \mid(Y \times Z)$ such that $\bar{\pi}^{*}(\rho)=\nu \times \omega$. It follows from Lemma 3.14 that $\rho \in \mathscr{J}(T, R)$. By weak stability $\rho=\mu \times \omega$, so that $T \times R \rightarrow S \times R$ is RUE. It follows that $T \rightarrow S$ is stable.

Even though stability is not an isomorphism invariant for extensions, the fact that weak stability is an isomorphism invariant means that to obtain a 
stable extension from a weakly stable one, we only need to construct an RUE model for its isomorphism class. By Proposition 3.2, this is always possible. An application of Theorem 2.2 yields the following.

Corollary 3.15. If $T \rightarrow S$ is a weakly stable extension then there exists an isomorphic extension $T^{\prime} \rightarrow S^{\prime}$ which is stable and topological.

\section{SOME LIFTING THEOREMS}

4.1. Mixing properties. Let $\mathscr{V}$ denote the group of all invertible bimeasurable measure preserving transformations of a Lebesgue space $(X, \mathscr{B}, \mu)$. Let $\mathscr{Z}$ denote the normal subgroup of Borel automorphisms equivalent to the identity. Then $\mathscr{U}=\mathscr{V} / \mathscr{K}$ is the group of equivalent measure preserving transformations of $(X, \mathscr{B}, \mu)$. An ergodic property $\mathscr{P}$ is an isomorphism invariant subset of $\mathscr{U}$ (i.e., a conjugacy class of $\mathscr{U}$ ), containing the set $\mathscr{E}$ of ergodic measure preserving transformations. If $T \in \mathscr{P}$ then we say $T$ has the property $\mathscr{P}$. A mixing property is an ergodic property which contains the weakly mixing transformations $\mathscr{W}$.

We are now ready to state and prove some lifting theorems. The general format for such a theorem will be the following:

Lifting Theorem. Suppose $S$ satisfies a mixing property $\mathscr{P}$ and $T \rightarrow S$ is an extension with some stability (i.e., maybe $T \rightarrow S$ is stable or self-stable). If $T$ is weakly mixing, then it also satisfies the property $\mathscr{P}$.

As we noted in the introduction, theorems of this type are well-known in the case of group extensions. We will see here how far these results extend to stable extensions.

4.2. Lifting theorems for partial mixing and its generalizations. Let $r \geq 2$ be fixed. Let $\mathbf{n}=\left(n^{1}, \ldots, n^{r}\right)$ be an $r$-tuple of integers and define $\|\mathbf{n}\|=$ $\min _{i \neq j}\left|n^{i}-n^{j}\right|$. We say a sequence $\left\{\mathbf{n}_{k}\right\}$ of $r$-tuples is expanding if $\left\|\mathbf{n}_{k}\right\| \rightarrow \infty$. Generally we denote a collection of expanding sequences of $r$-tuples by $\Sigma_{r}$, where for example, $\Sigma_{r}=Z^{r}$ will stand for the collection of all expanding sequences. In some cases it will also be convenient to let $\Sigma_{r}$ denote a single expanding sequence. Let $(X, \mathscr{B}, \mu, T)$ be an ergodic measure preserving transformation. We say $T$ is $r$-fold partially mixing on $\Sigma_{r}$ if for each $\left\{\mathbf{n}_{k}\right\} \in \Sigma_{r}$ and $A_{i} \in \mathscr{B}$ we have

$$
\limsup _{k \rightarrow \infty} \mu\left(T^{n_{k}^{1}} A_{1} \cap \cdots \cap T^{n_{k}^{r}} A_{r}\right) \geq \beta \mu\left(A_{1}\right) \cdots \mu\left(A_{r}\right),
$$

for some $\beta>0$. If $T$ is $r$-fold partially mixing on $\Sigma_{r}$, we define the mixing number $\alpha=m\left(T, \Sigma_{r}\right)$ to be the supremum of the numbers $\beta$ so that (13) holds for each $\left\{\mathbf{n}_{k}\right\} \in \Sigma_{r}$. We also define $m\left(T, \Sigma_{r}\right)=0$ if $T$ is not partially mixing, as for example if $T$ has pure point spectrum. Thus clearly $0 \leq \alpha \leq 1$. In the case $\alpha=1$ we say $T$ is $r$-fold mixing on $\Sigma_{r}$.

Proposition 4.1. The transformation $T$ is $r$-fold mixing on $\Sigma_{r}$ if and only if for every $\left\{\mathbf{n}_{k}\right\} \in \Sigma_{r}$ and $A_{i} \in \mathscr{B}$,

$$
\lim _{k \rightarrow \infty} \mu\left(T^{n_{k}^{1}} A_{1} \cap \cdots \cap T^{n_{k}^{r}} A_{r}\right)=\mu\left(A_{1}\right) \cdots \mu\left(A_{r}\right) .
$$

We will prove this in the next section. 
For $0<\alpha \leq 1$ and $\Sigma_{r}$ let us define

$$
\mathscr{M}\left(\Sigma_{r}, \alpha\right)=\left\{T \in \mathscr{U}: m\left(T, \Sigma_{r}\right) \geq \alpha\right\},
$$

and $\mathscr{M}\left(\Sigma_{r}, 0\right)=\left\{T \in \mathscr{U}: m\left(T, \Sigma_{r}\right)>0\right\}$. We now consider some well-known special cases:

(i) Let $\Sigma_{2}=\mathbf{Z}^{2}$. Then since $\mu\left(T^{n^{1}} A_{1} \cap T^{n^{2}} A_{2}\right)=\mu\left(T^{n^{1}-n^{2}} A_{1} \cap A_{2}\right)$, the property $\mathscr{M}\left(\Sigma_{2}, 0\right)$ is the property partial mixing (cf., [8, 9]). In particular, $m\left(T, \mathbf{Z}^{2}\right)=\alpha>0$ is $\alpha$-mixing for $T$ [9].

(ii) For $\Sigma_{2}=Z^{2}$ it follows from Proposition 4.1 that $\mathscr{M}\left(\Sigma_{2}, 1\right)$ is the property (ordinary) strong mixing (cf., [38]).

(iii) More generally for $r>2, \mathscr{M}\left(\mathbf{Z}^{r}, 1\right)$ is the property $r$-fold mixing (cf., [38]).

(iv) $\mathscr{M}\left(\mathbf{Z}^{r}, \alpha\right)$ is higher order partial mixing [7].

(v) For an increasing sequence $\left\{n_{k}\right\}$ of positive integers let $\Sigma_{2}=\left\{\left(0, n_{k}\right)\right\}$. Then $\mathscr{M}\left(\Sigma_{2}, 1\right)$ is the property mixing on the sequence $\left\{n_{k}\right\}$ [6].

In [6], it is noted that $T$ is weakly mixing if and only if there exists an increasing sequence $\left\{n_{k}\right\}$ such that $T$ is mixing on $\left\{n_{k}\right\}$. It then follows from a well-known result of Halmos and von Neumann that $T$ is weakly mixing if and only if it mixes on a sequence $\left\{n_{k}\right\}$ of density 1 . Clearly if $T \stackrel{\pi}{\rightarrow} S$ for some $S$ with discrete spectrum, then using sets of the form $A_{i} \in \pi^{-1} \mathscr{A} \subseteq \mathscr{B}$ in (13), we have $m\left(T, \Sigma_{r}\right)=0$ for any $\Sigma_{r}$. This proves the following lemma.

Lemma 4.2. For all $0 \leq \alpha \leq 1, \mathscr{M}\left(\Sigma_{r}, \alpha\right)$ is a mixing property.

Notice that if $T \rightarrow S$ and $T \in \mathscr{M}\left(\Sigma_{r}, \alpha\right)$ then $S \in \mathscr{M}\left(\Sigma_{r}, \alpha\right)$. The following lifting theorem shows that if there is enough stability the converse also holds.

Theorem 4.3. Suppose $S \in \mathscr{M}\left(\Sigma_{r}, \alpha\right)$. If $T \rightarrow S$ is an $r$-fold self-stable extension, so that in particular $T$ is weakly mixing, then $T \in \mathscr{M}\left(\Sigma_{r}, \alpha\right)$. In particular, $m\left(T, \Sigma_{r}\right)=m\left(S, \Sigma_{r}\right)$.

Corollary 4.4. The following properties lift to self-stable extensions: (i) partial mixing, (ii) strong mixing, (iii) r-fold mixing, (iv) $r$-fold partial mixing, and (v) mixing on a given collection of sequences.

4.3. The proofs. The proofs of Proposition 4.1 and Theorem 4.3 are based on the following lemma about self-joinings. The case $\Sigma_{1}=\mathbf{Z}$ appears in [21]. For an expanding sequence $\Sigma_{r}=\left\{\mathbf{n}_{k}\right\}$ of $r$-tuples, let $\mathscr{L}_{\Sigma_{r}} \Delta(T)$ denote the set of $r$-fold off-diagonal self-joinings of $T$ of the form

$$
\Delta_{n_{k}}\left(A_{1} \times A_{2} \times \cdots \times A_{r}\right)=\mu\left(T^{n_{k}^{1}} A_{1} \cap \cdots \cap T^{n_{k}^{r}} A_{r}\right) .
$$

The set $\mathscr{J}_{\Sigma_{r}}^{\Delta}(T)$ is given the subspace topology for the weak topology on the set of all joinings.

Lemma 4.5. For an expanding sequence $\Sigma_{r}$ of $r$-tuples, $m\left(T, \Sigma_{r}\right)=\alpha>0$ if and only if every limit point of $\mathscr{J}_{\Sigma_{r}}^{\Delta}(T)\left(\right.$ in $\left.\mathscr{J}_{r}(S)\right)$ is of the form

$$
\alpha \mu^{(r)}+(1-\alpha) \rho,
$$

where $\mu^{(r)}=\mu \times \cdots \times \mu$ and $\rho$ is an arbitrary $r$-fold self-joining of $T$ such that $\rho \perp \mu^{(r)}$. 
Proof. First we suppose that every limit point has the form (17). Then for any subsequence $\left\{\mathbf{n}_{k}^{\prime}\right\}$ of $\Sigma_{r}$ such that the sequence $\left\{\Delta_{\mathbf{n}_{k}^{\prime}}\right\}$ converges, we have

$$
\begin{aligned}
\lim _{k \rightarrow \infty} & \Delta_{\mathbf{n}_{k}^{\prime}}\left(A_{1} \times \cdots \times A_{r}\right) \\
& =\alpha \mu^{(r)}\left(A_{1} \times \cdots \times A_{r}\right)+(1-\alpha) \rho\left(A_{1} \times \cdots \times A_{r}\right) \\
& \geq \alpha \mu\left(A_{1}\right) \cdots \mu\left(A_{r}\right) .
\end{aligned}
$$

Thus (16) and (18) imply that there exists a subsequence $\left\{\mathbf{n}_{k}^{\prime}\right\}$ such that

$$
\begin{aligned}
\liminf _{k \rightarrow \infty} \mu\left(T^{n_{k}^{\prime \prime}} A_{1} \cap \cdots \cap T^{n_{k}^{\prime r}} A_{r}\right) & =\liminf _{k \rightarrow \infty} \Delta_{\mathbf{n}_{k}^{\prime}}\left(A_{1} \times \cdots \times A_{r}\right) \\
& \geq \alpha \mu\left(A_{1}\right) \cdots \mu\left(A_{r}\right),
\end{aligned}
$$

so that (13) holds.

Conversely, suppose $\liminf _{k \rightarrow \infty} \mu\left(T^{n_{k}^{1}} A_{1} \cap \cdots \cap T^{n_{k}^{r}} A_{r}\right) \geq \alpha \mu\left(A_{1}\right) \cdots \mu\left(A_{r}\right)$ for all $A_{i} \in \mathscr{B}$. Let $\xi$ be a limit point of $\mathcal{L}_{\Sigma_{r}}^{\Delta}(T)$. Then

$$
\begin{aligned}
\xi\left(A_{1} \times \cdots \times A_{r}\right) & \geq \liminf _{k \rightarrow \infty} \Delta_{\mathbf{n}_{k}}\left(A_{1} \times \cdots \times A_{r}\right) \\
& =\liminf _{k \rightarrow \infty} \mu\left(T^{n_{k}^{1}} A_{1} \cap \cdots \cap T^{n_{k}^{r}} A_{r}\right) \\
& \geq \alpha \mu\left(A_{1}\right) \cdots \mu\left(A_{r}\right) \\
& =\alpha \mu^{(r)}\left(A_{1} \times \cdots \times A_{r}\right) .
\end{aligned}
$$

Now $\rho^{\prime}=\xi-\alpha \mu^{(r)}$ is a priori a signed measure. However, by equation (19), $\rho^{\prime}\left(A_{1} \times \cdots \times A_{r}\right) \geq 0$ for all rectangles $A_{1} \times \cdots \times A_{r}$, so that $\rho^{\prime}$ is actually positive. It follows that $\rho^{\prime}=(1-\alpha) \rho$ for some joining $\rho$. Thus $\xi=\alpha \mu^{(r)}+(1-\alpha) \rho$.

Finally let us suppose that $\alpha<1$ and $\mu^{(r)}$ and $\rho$ are not mutually singular. Notice that $T$ is weakly mixing by Lemma 4.2 since $\alpha>0$, and thus $\mu^{(r)}$ is ergodic. By the ergodic decomposition theorem, we may assume without loss of generality that $\rho$ is also ergodic. Thus if $\mu^{(r)}$ and $\rho$ are not mutually singular they are equal and $\alpha=1$.

Proof of Proposition 4.1. If (13) holds for $\alpha=1$, it follows from Lemma 4.5 that product measure is the only limit point. This clearly implies that the limit (14) exists. The converse is obvious.

Proof of Theorem 4.3. It suffices to consider the case where $\Sigma_{r}$ consists of a single sequence, and $m\left(S, \Sigma_{r}\right)=\alpha>0$. We note that the natural factor map $\pi_{r}^{*}: \mathscr{L}_{r}(T) \rightarrow \mathscr{J}_{r}(S)$ is continuous. Let $\xi=\lim _{k \rightarrow \infty} \Delta_{k}$, i.e., a limit point for $\mathscr{J}_{\Sigma_{r}}^{\Delta}(T)$. Let $\Delta_{k}^{\prime}=\pi_{r}^{*}\left(\Delta_{k}\right)$. Clearly $\Delta_{k}^{\prime} \in \mathscr{J}_{\Sigma_{r}}^{\Delta}(S)$. It follows from the continuity of $\pi_{r}^{*}$ that the limit $\xi^{\prime}=\lim _{k \rightarrow \infty} \Delta_{k}^{\prime}$ exists and $\pi_{r}^{*}(\xi)=\xi^{\prime}$. Now since $m\left(S, \Sigma_{r}\right)=\alpha>0$ and $T \rightarrow S$ is $r$-fold self-stable, it follows from Lemma 4.5 that

$$
\xi^{\prime}=\alpha \nu^{(r)}+(1-\alpha) \rho^{\prime} .
$$

Let $\gamma$ and $\rho$ be any lifts of $\nu^{(r)}$ and $\rho^{\prime}$ to $\mathscr{F}_{r}(T)$ respectively. Since $\nu^{(r)}$ and $\rho^{\prime}$ are mutually singular, $\gamma$ and $\rho$ must also be. Furthermore, by $r$-fold self-stability $\gamma=\mu^{(r)}$. It follows that $\xi=\alpha \mu^{(r)}+(1-\alpha) \rho$ where $\mu^{(r)}$ and $\rho$ are mutually singular. The theorem now follows from Lemma 4.5.

4.4. Two more applications. We conclude this section by describing two more applications of 2-fold self-stability. Our first application is really a corollary of 
Theorem 4.3 in the case $\alpha=1$. Let $\sigma(T)$ denote the set of all sequences $\left\{n_{k}\right\}$ so that $T$ mixes on $\left\{n_{k}\right\}$. Clearly $\sigma(T)$ is an isomorphism invariant of $T$. By the comments preceding Lemma $4.2, \sigma(T) \neq \varnothing$ if and only if $T$ is weakly mixing. The invariant $\sigma(T)$ was studied by Friedman [6].

Corollary 4.6. If $S$ is weakly mixing and $T \rightarrow S$ is a 2-fold self-stable extension then $\sigma(T)=\sigma(S)$.

Our second application is the following. Blum and Hanson proved in [3] that an ergodic transformation $T$ is mixing if and only if for the mean ergodic theorem holds for every sequence $\left\{n_{k}\right\}$. More specifically, we say a sequence $\left\{n_{k}\right\}$ is a Blum-Hanson sequence if

$$
\lim _{N \rightarrow \infty}\left\|\frac{1}{N} \sum_{k=1}^{N} f \circ T^{n_{k}}-\int_{X} f d \mu\right\|_{2}=0,
$$

for all $f$ in $L^{2}(X, \mathscr{B}, \mu)$, where $\|\cdot\|_{2}$ denotes the $L^{2}$ norm. Thus $T$ is mixing if and only if every sequence is a Blum-Hanson sequence. Jones [19] showed that if $T$ is weakly mixing, every sequence of positive upper density is a Blum-Hanson sequence. The set of sequences for which (21) holds is clearly an isomorphism invariant for $T$. Let us denote this set of sequences by $\gamma(T)$. Friedman showed in [6] that (21) is equivalent to

$$
\lim _{N \rightarrow \infty} \frac{1}{N^{2}} \sum_{j, k=1}^{N} \mu\left(T^{n_{j}} A \cap T^{n_{k}} B\right)=\mu(A) \mu(B),
$$

for all $A, B \in \mathscr{B}$. Let $\mathscr{J}_{\left\{n_{k}\right\}_{2}}^{\mathrm{BH}}(T)$ denote the set of all joinings of the form

$$
\Theta_{N}(A \times B)=\frac{1}{N^{2}} \sum_{j, k=1}^{N} \mu\left(T^{n_{j}} A \cap T^{n_{k}} B\right) .
$$

Using the same methods as in the proofs of Lemma 4.5 and Theorem 4.3 we have the following:

Proposition 4.7. A sequence $\left\{n_{k}\right\}$ is a Blum-Hanson sequence for $T$ if and only if the only limit point for $\mathcal{F}_{\left\{n_{k}\right\}_{2}}^{\mathrm{BH}}(T)$ is the product joining $\mu \times \mu$.

Corollary 4.8. If $T \rightarrow S$ is a 2-fold self-stable extension with $S$ weakly mixing then $\left\{n_{k}\right\}$ is a Blum-Hanson sequence for $T$ if and only if it is a Blum-Hanson sequence for $S$.

\section{Natural Properties and the generalized Pinsker lemma}

The idea in this section is to try to exploit the following simple very general lifting theorem.

Proposition 5.1. Suppose $T \rightarrow S$ is a stable extension. If $S \perp R$ and $T \times R$ is ergodic then $T \perp R$.

Let $\mathscr{P}$ be an ergodic property in $\mathscr{U}$. We define the following related properties:

(i) Let $\mathscr{P}_{F}$ to be the set of all $S \in \mathscr{U}$ such that $T \rightarrow S$ for some $T \in \mathscr{P}$. 
(ii) Let $\mathscr{P} F=\left(\mathscr{P}_{F}\right)^{c}$, the complement of $\mathscr{P}_{F}$ in $\mathscr{U}$.

In other words, $\mathscr{P} F$ is the set of all $T$ with no factors in $\mathscr{P}$. Similar definitions are obtained by substituting disjointness for the absence factors.

(iii) Let $\mathscr{P}^{\perp}=\{R \in \mathscr{U}: R \perp T \forall T \in \mathscr{P}\}$.

Since disjointness is an isomorphism invariant it follows that $\mathscr{P} \perp$ is an ergodic property.

(iv) Define $\mathscr{P}_{\perp}=\left(\mathscr{P}^{\perp}\right)^{c}$.

Furstenberg's theorem, Theorem 3.9, implies that $\mathscr{P} \perp \subseteq \mathscr{P} F$. In general the converse is false, (cf., [30]). However, for certain kinds of transformations the converse is true.

\section{Definition 5.2.}

(i) A property $\mathscr{Q}$ such that $\mathscr{Q}^{F}=\mathscr{Q}^{\perp}$ is said to satisfy a generalized Pinsker lemma.

(ii) A property $\mathscr{P}$ is called a natural property if $\mathscr{P}=\mathscr{Q}^{F}=\mathscr{Q}^{\perp}$, where $\mathscr{Q}$ satisfies a generalized Pinsker lemma.

Recall that a measure preserving system $T$ is said to have the $K$-property, denoted $\mathscr{K}$, if it has no factors with entropy zero. In particular, if $\mathscr{Z}$ denotes the set of all ergodic transformations with entropy zero, then $\mathscr{K}=\mathscr{Z}^{F}$. The following lemma is called the Pinsker lemma, (cf., [36] for a proof).

Lemma 5.3 (Pinsker [27]). $\mathscr{K}=\mathscr{Z}^{F}=\mathscr{Z}^{\perp}$.

In particular, $\mathscr{K}$ is a natural property.

There are several other well-known examples of natural properties and corresponding generalized Pinsker lemmas. Furstenberg [10] showed that the set $\mathscr{D}$ of transformations with discrete spectrum satisfies $\mathscr{D}^{\perp}=\mathscr{D}^{F}$, and so weak mixing $\mathscr{W}=\mathscr{D}^{F}$ is a natural property. P. Walters [39] studies rigidity, denoted here by $\mathscr{R}$, and showed that mild mixing $\mathscr{M}=\mathscr{R}^{F}$ (cf., [14]) is a natural property since $\mathscr{M}=\mathscr{R}^{F}=\mathscr{R}^{\perp}$. We will discuss Walters' results in greater detail below. Because of Proposition 5.1, natural properties satisfy the following lifting theorem.

Proposition 5.4. Let $\mathscr{P}$ be a natural property and suppose $S$ is weakly mixing and satisfies $\mathscr{P}$. Let $T \rightarrow S$ be a stable extension. If $T$ is weakly mixing then it also satisfies the property $\mathscr{P}$.

Corollary 5.5. The Lifting Theorem holds in the case of stable extensions for mild mixing and the $K$-property.

K. Berg [1] proved the group extension case of Theorem 5.1 for the more general notion of quasidisjointness. Walters [39] proved the Lifting Theorem (Corollary 5.5) for mild mixing to group extensions. The fact that the $K$ property lifts to group extensions is first proved in [26]. RUE based proofs of the Lifting Theorem for the $K$-property appear in [5 and 29].

5.1. Sequence rigidity and Walters' Pinsker lemma. In this section we look more closely at Walters' results [39]. Let $\Sigma$ denote a nonempty collection of increasing sequences. Let $\mathscr{R}(\Sigma)$ denote the set of measure preserving systems $T$ such that for all sequences $\left\{n_{k}\right\} \in \Sigma, T^{n_{k}} \rightarrow I$ in the strong operator topology on $L^{2}(X, \mathscr{B}, \mu)$; that is:

$$
\lim _{k \rightarrow \infty}\left\|f \circ T^{n_{k}}-f\right\|_{2}=0 .
$$

We define $\mathscr{M}(\Sigma)=(\mathscr{R}(\Sigma))^{F}$. 
Proposition 5.6 (Walters [39]). For any nonempty collection $\Sigma$ of sequences, the property $\mathscr{R}(\Sigma)$ satisfies a generalized Pinsker lemma. Thus $\mathscr{M}(\Sigma)=(\mathscr{R}(\Sigma))^{F}=$ $(\mathscr{R}(\Sigma))^{\perp}$ is a natural property.

Walters' proof of Proposition 5.6 depends on spectral theory. A different proof will be given after Theorem 5.12 below.

Corollary 5.7. The properties $\mathscr{M}(\Sigma)$ satisfy the Lifting Theorem in the case of weakly mixing stable extensions.

Walters obtained this result for totally ergodic group extensions [39].

5.2. A natural property which is not a mixing property. The following proposition provides a counterexample to the possible conjecture that natural properties always involve 'more' mixing.

Proposition 5.8. The Bernoulli property $\mathscr{B}$ satisfies a generalized Pinsker lemma. In particular, entropy zero $\mathscr{Z}$ is a natural property.

Proof. We have $\mathscr{B}^{\perp} \subseteq \mathscr{B}^{F}=\mathscr{Z}$ by Sinai's theorem. Also $\mathscr{B} \subseteq \mathscr{K}=\mathscr{Z}^{\perp}$. Since by a general result [10], $\mathscr{Z} \subseteq \mathscr{Z}^{\perp \perp}$, it follows that $\mathscr{Z} \subseteq \mathscr{B}^{\perp}$.

Corollary 5.9. If $S$ has entropy zero and $T \rightarrow S$ is a stable extension of $S$ then $T$ has entropy zero.

5.3. Quasifactors and generalized Pinsker lemmas. In this section we consider generalized Pinsker lemmas in terms Glasner's theory of quasifactors [16].

Let $(X, \mathscr{B}, \mu, T)$ be an ergodic topological dynamical system. Then $X^{*}$ is compact and completely metrizable in the weak-* topology and $\mathscr{B}^{*}$ is the corresponding Borel structure. Let $T^{*}$ denote the homeomorphism (4) of $X^{*}$ induced by $T$ on $X$, so that in particular,

$$
\int_{X}(f \circ T) d \theta=\int_{X} f d\left(T^{*} \theta\right),
$$

for all $\theta \in X^{*}$ and $f \in C(X)$, where $C(X)$ denotes the set of real-valued continuous functions on $X$. Given $f \in C(X)$ we define $f^{*} \in C\left(X^{*}\right)$ by

$$
f^{*}(\theta)=\int_{X} f d \theta .
$$

It follows that

$$
(f \circ T)^{*}=f^{*} \circ T^{*}
$$

and

$$
\left|f^{*}(\theta)\right| \leq|f|^{*}(\theta) .
$$

Let $X^{* *}$ denote the set of all probability measures on $\left(X^{*}, \mathscr{B}^{*}\right)$. The set of $T^{*}$ invariant measures on $X^{* *}$ will be denoted by $X_{T}^{* *}$. Any $\lambda \in X_{T}^{* *}$ defines a measure preserving dynamical system $\left(X^{*}, \mathscr{B}^{*}, \lambda, T^{*}\right)$. A measure $\lambda \in X^{* *}$ satisfies the barycenter condition [16] if for all $f \in C(X)$,

$$
\int_{X} f d \mu=\int_{X^{*}} f^{*} d \lambda
$$

A dynamical system $\left(X^{*}, \mathscr{B}^{*}, \lambda, T^{*}\right)$ which is ergodic and such that $\lambda$ satisfies (27) is called a quasifactor [16] of $(X, \mathscr{B}, \mu, T)$. Glasner proves the following results about quasifactors: 
Theorem 5.10 (Glasner, [16]).

(i) Up to isomorphism the quasifactors of $T$ depend only on the isomorphism class of $T$.

(ii) For every factor $S$ of a measure preserving system $T$ there is a canonically associated isomorphic quasifactor $S^{\prime}$.

(iii) Two systems $T$ and $R$ are disjoint if and only if $T$ has no factor $S$ isomorphic to any nontrivial quasifactor $Q$ of $S$.

We say that a property $\mathscr{Q}$ closed under quasifactorization if $\mathscr{Q}_{Q F}=\mathscr{Q}$, where $\mathscr{Q}_{Q F}$ denotes the set of transformations isomorphic to a quasifactor of some $T \in \mathscr{Q}$.

Proposition 5.11. A property $\mathscr{Q}$ which is closed under quasifactorization satisfies a generalized Pinsker lemma.

Proof. Suppose $\mathscr{Q}=\mathscr{Q}_{Q F}$. If $T \in \mathscr{Q}^{F}$ and $T \rightarrow S$ then $S \notin \mathscr{Q}$. For any $P \in \mathscr{Q}$ and quasifactor $R$ of $P, R \in \mathscr{Q}$. This implies that $R$ and $S$ are not isomorphic, (since $\mathscr{Q}$ is closed under conjugation). Then by Theorem 5.10, $T \perp P$, so $T \in \mathscr{Q}^{\perp}$. Thus $\mathscr{Q}^{F} \subseteq \mathscr{Q}^{\perp}$ and $\mathscr{Q}$ is natural.

5.4. Quasifactors of sequence rigid transformations. In this section we reconsider the sequence rigidity properties $\mathscr{R}(\Sigma)$.

Theorem 5.12. For any $\Sigma \neq \varnothing$, the property $\mathscr{R}(\Sigma)$ is closed under quasifactorization.

Before proving the theorem we prove some lemmas.

Lemma 5.13. If $\left(X^{*}, \mathscr{B}^{*}, \lambda, T^{*}\right)$ is a quasifactor of $(X, \mathscr{B}, \mu, T)$ then for every $f \in C(X)$,

$$
\int_{X^{*}}\left|f^{*} \circ\left(T^{*}\right)^{n}-f^{*}\right| d \lambda \leq \int_{X}\left|f \circ T^{n}-f\right| d \mu .
$$

Proof. This follows from (25), (26), and (27).

Let $A\left(X^{*}\right)$ denote the algebra of functions generated by the functions of the form $f^{*}$ for $f \in C(X)$.

Lemma 5.14. The set $A\left(X^{*}\right)$ is dense in $L^{1}\left(X^{*}, \mathscr{B}^{*}, \lambda\right)$.

Proof. The functions $g \in C\left(X^{*}\right)$ are dense in $L^{1}\left(X^{*}, \mathscr{B}^{*}, \lambda\right)$. Thus it suffices to show that $A\left(X^{*}\right)$ is uniformly dense in $C\left(X^{*}\right)$, (since uniform convergence implies $L^{1}$ convergence). We have $1 \in A\left(X^{*}\right)$ since $1=1^{*}$, and $A\left(X^{*}\right)$ separates points since the functions $f^{*}$ do. The lemma now follows from the Stone Weierstrass Theorem.

Proof of Theorem 5.12. It suffices to prove the case where $\Sigma$ consists of a single sequence. We suppose $R \in \mathscr{R}(\Sigma)$ and show that $R^{*}$ is also $\Sigma$-rigid. Using the Hölder inequality, it suffices to show that for any $\varepsilon$ and for all $f \in L^{1}\left(X^{*}, \mathscr{B}^{*}, \lambda\right)$ we have

$$
\left\|f \circ\left(R^{*}\right)^{n}-f\right\|_{1}<\varepsilon,
$$

for infinitely many $n \in \Sigma$. By Lemma 5.14 , for any $f \in L^{1}\left(X^{*}, \mathscr{B} *, \lambda\right)$ there exists $F \in A\left(X^{*}\right)$ such that $\|f-F\|_{1}<\varepsilon / 3$. Since $\lambda$ is $R^{*}$ invariant, we have $\left\|f \circ\left(R^{*}\right)^{n}-F \circ\left(R^{*}\right)^{n}\right\|_{1}<\varepsilon / 3$, so that

$$
\left\|f \circ\left(R^{*}\right)^{n}-f\right\|_{1}<2 \varepsilon / 3+\left\|F \circ\left(R^{*}\right)^{n}-F\right\|_{1} .
$$


Now since $F \in A\left(X^{*}\right)$, there exist $g_{k, l} \in C(X), k=1, \ldots, m, l=1, \ldots$, $r_{m}$, such that

$$
F(\theta)=\sum_{k=1}^{m} g_{k, 1}^{*}(\theta) \cdots g_{k, r_{k}}^{*}(\theta)
$$

Then

$$
\begin{aligned}
\left\|F \circ\left(R^{*}\right)^{n}-F\right\| & \leq \sum_{k=1}^{m}\left\|\prod_{l=1}^{r_{k}} g_{k, l}^{*} \circ\left(R^{*}\right)^{n}-\prod_{l=1}^{r_{k}} g_{k, l}^{*}\right\|_{1} \\
& \leq \sum_{k=1}^{m} \sum_{l=1}^{r_{k}} c_{k, l}\left\|g_{k, l}^{*} \circ\left(R^{*}\right)^{n}-g_{k, l}^{*}\right\|_{1} \\
& \leq K \sum_{k=1}^{m} \sum_{l=1}^{r_{k}}\left\|g_{k, l}^{*} \circ\left(R^{*}\right)^{n}-g_{k, l}^{*}\right\|_{1} .
\end{aligned}
$$

Letting $L=K \Sigma r_{k}$ and $\varepsilon_{1}=\varepsilon / 3 L$, choose $n_{0}$ so large that for all $k, l$,

$$
\left\|g_{k, l}^{*} \circ\left(R^{*}\right)^{n}-g_{k, l}^{*}\right\|_{1}<\varepsilon_{1} .
$$

(29) then follows from (30) and (32).

An easy corollary of Theorem 5.12 is a new proof of Walters' Pinsker Lemma, Theorem 5.6.

Proof of Theorem 5.6. This follows from Proposition 5.11 and Theorem 5.12.

\section{PART II. EXAMPles OF STABle EXTENSIONS}

\section{STABILITY FOR DISTAL EXTENSIONS}

Distal extensions provide a natural generalization of the notion of a group extension. The general theory of distal extensions was independently studied by Furstenberg [12], (cf., also [13]), and Zimmer [42, 43], (who used the term 'generalized discrete spectrum extension' instead of 'distal extension'). An important result in the theory is that distal extensions are built out of isometric extensions, a kind of extension closely related to group extensions.

6.1. Isometric extensions. Let $G$ be a compact metric group, let $H$ be a closed subgroup of $G$, and let $G / H$ be the corresponding homogeneous space. Let $h^{g}$ denote the natural transitive, measure preserving, isometric action of $G$ on $(G / H, \mathscr{C}, \omega), h^{g}\left(g_{1} H\right)=g g_{1} H$, where $\mathscr{C}$ is the standard Borel structure on $G / H$ and $\omega$ is the projection of Haar measure. For a measure preserving transformation $(Y, \mathscr{A}, \nu, S)$ and cocycle $\varphi: Y \rightarrow G$ the corresponding action extension (6) is given by

$$
T(y, g H)=(S y, \varphi(y) g H),
$$

and is called an isometric extension.

There is a well-known alternative characterization of isometric extensions. Suppose $h^{g}$ is a free transitive action of a locally compact group $G$ on a compact metric space $Z$ such that for each $g \in G, h^{g}$ is an isometry. In particular 
this implies that $G$ is compact and $h^{g}$ preserves a probability measure $\omega$ on $(Z, \mathscr{C})$, where as usual $\mathscr{C}$ is the standard Borel structure on $Z$. For any measure preserving system $(Y, \mathscr{A}, \nu, S)$ and cocycle $\varphi^{\prime}: Y \rightarrow G$, let

$$
T^{\prime}(y, z)=\left(S y, h^{\varphi^{\prime}(y)} z\right)
$$

be the corresponding action extension.

Lemma 6.1. If the action extension (34) satisfies the conditions above then there exists a closed subgroup $H$ of $G$ and a cocycle $\varphi: Y \rightarrow G$ so that the extension $T \rightarrow S$ defined by (33) is isomorphic to the extension $T^{\prime} \rightarrow S$ defined by (34).

Proposition 6.2. If $T \rightarrow S$ is an ergodic isometric extension then $T \rightarrow S$ is RUE and stable.

The proof that $T \rightarrow S$ is RUE follows from Lemma 3.1 and the next lemma. The proof of stability is the same as the proof of Corollary 3.8 .

Lemma 6.3 (Zimmer [42]). If $T \rightarrow S$ is an ergodic isometric extension then there exists an ergodic compact group extension $U \rightarrow S$ such that $U \rightarrow T \rightarrow S$.

6.2. Distal extensions. An extension is a distal extension if it is isomorphic to an extension built up by induction as follows (cf., [43]):

(i) An isometric extension is distal.

(ii) If $U \rightarrow T$ and $T \rightarrow S$ are distal then the composition $U \rightarrow S$ is distal.

(iii) If $T_{i+1} \rightarrow T_{i}$ are distal and $T \rightarrow T_{i}$ for all $i \in \mathbf{N}$ then $T \rightarrow T_{1}$ is distal.

In the case (3) $T$ is called an inverse limit of the sequence $T_{i}$.

Lemma 6.4. Distal extensions are RUE and stable.

This follows from [29] where it is shown that RUE extends to inverse limits of RUE extensions and from the definition of stability in terms of RUE.

6.3. Affine extensions. In this section we consider a different generalization of group extensions.

Starting with the same set up as in (7), we let $\alpha$ be a continuous automorphism of $G$. An $\alpha$-affine extension is a skew product of the form

$$
T(y, g)=(S y, \varphi(y) \alpha(g)) .
$$

Note that since $\alpha$ preserves $\omega, \nu \times \omega$ is $T$ invariant. The following theorem extends a result in [29].

Theorem 6.5. Let $T \rightarrow S$ be an ergodic $\alpha$-affine extension. Let $h(\alpha)$ denote the topological entropy of $\alpha$. The following are equivalent:

(i) $h(\alpha)=0$.

(ii) $T \rightarrow S$ is a distal extension.

(iii) $T \rightarrow S$ is $R U E$ and stable.

Proof. First we observe that, as in the group extension case, RUE is equivalent to stability for $\alpha$-affine extensions. In [28] we showed that (i) is equivalent to (iii) using an argument based on arguments of Walters, [39]. Thus (i) is equivalent to (iii). That (ii) implies (i) is well known: $h(\alpha)=h(T \mid S)$, the relative entropy of the extension, and $h(T \mid S)=0$ for distal extensions. Thus it suffices to show that (i) implies (ii). The proof is based on further arguments from [39]. 
Walters shows in [40] that an arbitrary $\alpha$-affine extension $T \rightarrow S$ with $h(\alpha)=0$ factors into an inverse limit of three special types of $\alpha$-affine extensions:

1. $G$ is a compact abelian and $\alpha^{n}$ is unipotent for some $n$,

2. $G$ is finite, and

3. $G$ is a compact simple Lie group.

Thus it suffices to show that extensions of each of these three types are distal.

Type 1. Further arguments in [40] show that an extension $T \rightarrow S$ of this type factors into series of extensions of the following more special type:

$1^{\prime}$. $G$ is compact abelian and $\alpha$ is unipotent.

Thus it suffices to consider extensions $T \rightarrow S$ of the type $1^{\prime}$. Note that $\alpha$ is unipotent if $\alpha^{k}=$ Id for some $k$. Thus we have

$$
\begin{aligned}
T^{k}(y, z) & =\left(S^{k} y, \varphi\left(S^{k-1} y\right)(\alpha \circ \varphi)\left(S^{k-2} y\right) \cdots\left(\alpha^{k-1} \circ \varphi\right)(y) \alpha^{k}(z)\right) \\
& =\left(S^{k} y, \varphi^{\prime}(y) \alpha^{k}(z)\right)=\left(S^{k} y, \varphi^{\prime}(y)(z)\right),
\end{aligned}
$$

which implies that $T^{k} \rightarrow S^{k}$ is a group extension and thus distal. It follows using Zimmer's characterization of distal extensions in terms of separating sieves [43], that $T \rightarrow S$ is a distal extension.

Type 2. Every finite extension is distal.

Type 3. In this case there exists $k$ so that $\alpha^{k}$ is an inner automorphism, [40]. Thus there exists $h \in G$ such that $\alpha^{k}(z)=h^{-1} z h$. By (35) we have

$$
T^{k}(y, z)=\left(S^{k}, \varphi^{\prime}(y) h^{-1} z h\right) .
$$

Letting $W_{y} z=\varphi^{\prime}(y) h^{-1} z h$, we observe that $W_{y}$ is an isometry for all $y$. It follows from Lemma 6.1 that $T^{k} \rightarrow S^{k}$ is an isometric extension and thus distal, so that $T \rightarrow S$ is a distal extension.

\section{THE RELATIVE ENTROPY OF STABLE EXTENSIONS}

The purpose of this section is to prove the following general result, already suggested by Corollary 5.9 and Theorem 6.5 .

Theorem 7.1. If $T \rightarrow S$ is stable or 2-fold self-stable then $T \rightarrow S$ has relative entropy zero.

Let $h(T \mid S)$ denote the relative entropy of the extension $T \rightarrow S$, (cf. [35]). If $h(T \mid S)>0$, then by Thouvenot's Relative Sinai Theorem [35] there exists an isomorphic extension $T^{\prime} \rightarrow S^{\prime}$ with $T^{\prime} \rightarrow B \times S^{\prime} \rightarrow S^{\prime}$, where $B$ is a Bernoulli shift of entropy $h=h(T \mid S)$. This reduces Theorem 7.1 to the next lemma.

Theorem 7.2. The extension $S \times R \rightarrow S$ is not 2-fold self-stable. If $R$ is weakly mixing, the extension $S \times R \rightarrow S$ is not stable.

Proof. Assume without loss of generality that $S \times R$ is weakly mixing on $Y \times Z$. Then

$$
(S \times R) \times(S \times R)=(S \times S) \times(R \times R) \rightarrow S \times S,
$$

is not RUE since by Lemma $3.10 R \times R$ is not uniquely ergodic.

Similarly in the second case, since $R$ is weakly mixing it suffices to show that $(S \times R) \times R=S \times(R \times R) \rightarrow S \times R$ is not RUE. Clearly $\nu \times \omega \times \omega \in$ 
$(Y \times(Z \times Z))^{*} \mid Y \times Z$, and we claim that also $\nu \times \Delta_{R^{1}} \in(Y \times(Z \times Z))^{*} \mid Y \times Z$. But this just amounts to

$$
\begin{aligned}
\left(\nu \times \Delta_{R^{1}}\right)(E \times F \times Z) & =\omega(E) \Delta_{R^{1}}(F \times Z)=\omega(E) \nu(F \cap Z) \\
& =\omega(E) \nu(F)=(\omega \times \nu)(E \times F) .
\end{aligned}
$$

\section{A STABLE EXTENSION WHICH IS NOT DISTAL}

Throughout this section $(X, \mathscr{B}, \mu, T)$ and $(Y, \mathscr{A}, \nu, S)$ will denote topological dynamical systems such that $\pi: X \rightarrow Y$ is a continuous measure preserving factor map. We say $T$ is a topological extension of $S$. For a continuous ergodic flow $\left(Z, \mathscr{C}, \omega, h^{s}\right)$ on a compact metric space, and $\varphi \in C(Y)$, we let $X=Y \times Z$ and define the flow extension

$$
T_{\varphi}(y, z)=\left(S y, h^{\varphi(y)} z\right) .
$$

Notice that $T_{\varphi}$ is a product measure preserving homeomorphism. We denote the set of all (additive) coboundaries $\varphi(y)=\psi(S y)-\psi(y)$ with continuous transfer function $\psi$ by $\mathscr{B} \subseteq C(Y)$, and denote the uniform closure of $\mathscr{B}$ by $\overline{\mathscr{B}}$. Notice that if $\varphi \in \overline{\mathscr{B}}$ then $\int_{Y} \varphi d \nu=0$, a fact which remains true if $\nu$ is replaced any other $S$ invariant measure. In general $\mathscr{B}$ is not closed. The following result is due to Glasner and Weiss [18].

Theorem 8.1. Suppose $S$ is strictly ergodic and let $h^{s}$ be a continuous weakly mixing flow. Then there exists a dense $G_{\delta}$ subset $\mathscr{R}_{0} \subseteq \overline{\mathscr{B}}$ such that if $\varphi \in \mathscr{R}_{0}$, the corresponding flow extension $T_{\varphi}$ is a relatively weakly mixing extension over $S$.

We say that the "generic" flow extension by a weakly mixing flow is relatively weakly mixing. In particular, Theorem 8.1 says that generically $T_{\varphi}$ is not a distal extension of $S$.

Now for an arbitrary topological extension $(X, \mathscr{B}, \mu, T)$ of $(Y, \mathscr{A}, \nu, S)$ let us consider the homeomorphism $T^{*}$ induced by $T$ on $X^{*}$. Clearly $X^{*} \mid Y$ is a closed $T^{*}$ invariant subset of $X^{*}$, which contains the fixed point $\mu$. Associated with this fixed point is the $T^{*}$ invariant measure $\delta_{\mu}$, i.e. the point mass at $\mu$. In [18], Glasner and Weiss studied the situation in which this is the unique invariant measure-that is, when $T^{*}$ is uniquely ergodic on $X^{*} \mid Y$. We will call this property of $T \rightarrow S$ the $G W$-property. The following result relates the $\mathrm{GW}$-property to stability.

Proposition 8.2. If a topological extension $T$ of $S$ has the $G W$-property then it is a stable extension.

The proof is very similar to the proof of Proposition 2.1 of [18].

Proof. Let $T \stackrel{\pi}{\rightarrow} S$ be a topological extension with the GW-property. Clearly the GW-property implies RUE, and thus by Proposition 3.13, it suffices to show that $T \stackrel{\pi}{\rightarrow} S$ is weakly stable.

Suppose $(W, \mathscr{C}, \omega, R)$ is such that $T \times R$ is ergodic. Let $\bar{\pi}(x, w)=$ $(\pi(x), w)=(y, w)$, so that $T \times R \stackrel{\bar{\pi}}{\rightarrow} S \times R$. Let $\rho$ be a joining of $T$ and $R$ so that $\bar{\pi}^{*}(\rho)=\nu \times \omega$. We need to show that $\rho=\mu \times \omega$.

By Lemma 2.1 we have

$$
\rho=\int_{W} \rho_{w} d \omega(w)
$$


Projecting $\rho$ to $S \times R$ we have

$$
\bar{\pi}^{*}(\rho)=\int_{W} \pi^{*} \rho_{w} d \omega(w) .
$$

Since $\bar{\pi}^{*}(\rho)=\nu \times \omega$, the uniqueness of the decomposition (39) implies that $\pi^{*}\left(\rho_{w}\right)=\nu$ for $\omega$ a.e. $w$. Thus we can define a map $\zeta: W \rightarrow X^{*} \mid Y$ by $\zeta(w)=\rho_{w}$ and let $\lambda=\zeta^{*}(\omega)$. The $T \times R$ invariance of $\rho$ implies that $T^{*} \rho_{w}=\rho_{R_{w}}$, from which it follows that $T^{*} \circ \zeta=\zeta \circ R$. Thus $\lambda$ is a $T^{*}$ invariant measure on $X^{*} \mid Y$. By the GW-property for $T \stackrel{\pi}{\rightarrow} S, \lambda=\delta_{\mu}$. This implies that $\rho_{w}=\zeta(w)=\mu$ for $\omega$ a.e. $w$, and by (38) we have $\rho=\mu \times \omega$.

Note. $\left(X^{*}, \mathscr{B}^{*}, \lambda, T^{*}\right)$ is a quasifactor of $T$.

The difference between the GW-property and stability is not very great. The $\mathrm{GW}$-property requires that the product joining lift uniquely from $S$ to $T$ whether or not it is ergodic, but stability only requires that the product joining lift uniquely if it is ergodic.

Theorem 8.3 (Glasner and Weiss [18]). Let $S$ be an irrational rotation on the circle $Y$ and let $h^{s}$ be a strictly ergodic flow on $Z$. Then there exists a dense $G_{\delta}$ subset $\mathscr{R}_{1} \subseteq \overline{\mathscr{B}}$ such that if $\varphi \in \mathscr{R}_{1}$ then the corresponding flow extension $T_{\varphi}$ has the $G W$-property.

In particular, Theorem 8.3 provides a negative answer to the question "is every stable extension distal?" by showing that the generic flow extension over an irrational rotation is stable but not distal. This follows from Proposition 8.2 and Theorem 8.1. However, Theorem 8.3 is somewhat unsatisfactory from our point of view because it fails to produce any new lifting theorems; the extensions produced are never weakly mixing. Our main result in this section corrects this problem. It is a generalization of Theorem 8.3 to the case where $S$ is weakly mixing:

Theorem 8.4. Let $(Y, \mathscr{A}, \nu, S)$ be a weakly mixing homeomorphism and let $\left(Z, \mathscr{C}, \omega, h^{s}\right)$ be a continuous uniquely ergodic flow. Then there exists a dense $G_{\delta}$ subset $\mathscr{R} \subseteq \overline{\mathscr{B}}$ such that for $\varphi \in \mathscr{R}$ the corresponding flow extension $T_{\varphi}$ has the GW-property.

Corollary 8.5. If $S$ and $h^{s}$ are both strictly ergodic and weakly mixing then the generic flow extension $T_{\varphi}$ is stable and relatively weakly mixing. In particular, there exist stable extensions of any weakly mixing transformation which are not distal.

Proof of Corollary 8.5. Since distal extensions are not relatively weakly mixing, the second statement follows from the first using the Jewett-Krieger Theorem to insure that $S$ is strictly ergodic.

The first statement follows from Theorems 8.1 and 8.4.

The proof of Theorem 8.4 takes up most of the remainder of the paper. Our approach closely follows the powerful method developed by Glasner and Weiss in [17] and [18]. We begin with the following ergodic theorem.

Lemma 8.6. Let $R$ be an ergodic homeomorphism of a compact metric space $V$ preserving a probability measure $\gamma$. Let $W$ be another compact metric space, 
and let $\theta \in(V \times W)^{*} \mid V$. Then for any $\phi \in C(V \times V \times W)$,

$$
\begin{aligned}
& \lim _{n \rightarrow \infty} \frac{1}{n+1} \sum_{j=0}^{\infty} \int_{V \times W} \phi\left(R^{j} v, v, w\right) d \theta(v, w) \\
& \quad=\int_{V \times W} \int_{V} \phi\left(v_{1}, v_{2}, w\right) d \mu\left(v_{1}\right) d \theta\left(v_{2}, w\right) .
\end{aligned}
$$

Proof. Let $\theta=\int_{V} \theta_{v} d \gamma(v)$ be the disintegration of $\theta$ over $\gamma$, (Lemma 2.1). Let $\Delta_{R^{n}}$ denote the $n$th off diagonal measure (11) on $V \times V$, and let

$$
\Sigma_{n}=\frac{1}{n+1} \sum_{k=0}^{n} \Delta_{R^{k}} .
$$

Let $\rho_{n}=\int_{V \times V} \theta_{v_{2}} d \Sigma_{n}\left(v_{1}, v_{2}\right)$ and $\rho=\int_{V \times V} \theta_{v_{2}} d(\mu \times \mu)\left(v_{1}, v_{2}\right)$. Then (40) is equivalent to the weak-* convergence of $\rho_{n}$ to $\rho$, and it suffices to prove (40) for a set $\mathscr{F}$ of functions with a dense span in $C(V \times V \times W)$. In particular, by the Stone-Weierstrass Theorem we can take $\mathscr{F}$ to be the set of all functions of the form $\phi\left(v_{1}, v_{2}, w\right)=f\left(v_{1}\right) g\left(v_{2}\right) h(w)$, for $f, g \in C(V)$ and $h \in C(W)$.

We have

$$
\begin{aligned}
\int_{V \times V \times W} f\left(v_{1}\right) g\left(v_{2}\right) h(w) d \rho_{n}\left(v_{1}, v_{2}, w\right) \\
=\int_{V \times V} f\left(v_{1}\right) g\left(v_{2}\right) \int_{W} h(w) d \theta_{v_{2}}(w) d \Sigma_{n}\left(v_{1}, v_{2}\right) \\
=\int_{V \times V} f\left(v_{1}\right) \tilde{g}\left(v_{2}\right) d \Sigma_{n}\left(v_{1}, v_{2}\right),
\end{aligned}
$$

where

$$
\tilde{g}\left(v_{2}\right)=g\left(v_{1}\right) \int_{W} h(w) d \theta_{v_{2}}(w) .
$$

Now since $R$ is ergodic and $f, \tilde{g} \in L^{2}(V, \gamma)$, we have

$$
\begin{aligned}
\lim _{n \rightarrow \infty} & \int_{V \times V} f\left(v_{1}\right) \tilde{g}\left(v_{2}\right) d \Sigma_{n}\left(v_{1}, v_{2}\right) \\
& =\lim _{n \rightarrow \infty} \frac{1}{n+1} \sum_{K=0}^{\infty} \int_{V} f\left(R^{n} v\right) \tilde{g}(v) d \mu(v) \\
& =\int_{V \times V} f\left(v_{1}\right) \tilde{g}\left(v_{2}\right) d(\mu \times \mu)\left(v_{1}, v_{2}\right) \\
& =\int_{V \times V} f\left(v_{1}\right) g\left(v_{2}\right) \int_{W} h(w) d \theta_{v_{2}}(w) d(\mu \times \mu)\left(v_{1}, v_{2}\right) .
\end{aligned}
$$

When combined with (41), this completes the proof.

Lemma 8.7. Let $Y$ be a compact metric space with a nonatomic probability measure $\nu$ on $Y$. Let $P \subseteq Y$ be a Cantor set, (i.e., closed, perfect, and totally disconnected) with $\nu(P)>0$, and let $L>0$. If $\left.\nu\right|_{P}$ is the restriction of $\nu$ to $P$, then there exists a continuous map $\tau: P \rightarrow[0, L]$, such that

$$
\tau^{*}(\nu \mid P)=d s / L
$$

where $d s / L$ denotes normalized Lebesgue measure on the interval $[0, L]$. 
This follows easily from the fact that any two Cantor sets are homeomorphic (cf., [24]).

Let $F \in C\left(X^{*} \mid Y\right), \varepsilon>0$, and define

$$
E_{F, \varepsilon}=\left\{\varphi \in \mathscr{B}:\left\|\frac{1}{n+1} \sum_{k=0}^{n} F\left(T_{\varphi}^{* k} \theta\right)-F(\nu \times \omega)\right\|<\varepsilon \text { for some } n \geq 1\right\},
$$

where $\|\cdot\|$ denotes the sup norm on $C\left(X^{*} \mid Y\right)$. Notice that $\varphi \in \mathscr{B}$ implies $\varphi=\psi \circ S-\psi$, for $\psi \in C(Y)$. Clearly $E_{F, \varepsilon}$ is open in $C(Y)$.

Let $\left\{F_{k}\right\} \subseteq C\left(X^{*} \mid Y\right)$ be an arbitrary countable collection of continuous real-valued functions with a dense linear span in $C\left(X^{*} \mid Y\right)$. Let

$$
\mathscr{R}=\bigcap_{k=1}^{\infty} \bigcap_{j=1}^{\infty} E_{F_{k}, 1 / j}
$$

Clearly $\mathscr{R}$ is $G_{\delta}$, (although possibly empty a priori). Since the set of functions for which ergodic averages converge uniformly is a closed subspace of $C\left(X^{*} \mid Y\right)$, it follows that for any $\varphi \in \mathscr{R}$ the corresponding extension $T_{\varphi}$ of $S$ has the GW-property. We will complete the proof of Theorem 8.4 using the Baire Category Theorem to show that $\mathscr{R}$ is dense in $\mathscr{B}$. In particular we will show that for a suitable choice of functions $\left\{F_{k}\right\}, \bar{E}_{F_{k}, \varepsilon}=\overline{\mathscr{B}}$ for each $k$.

First we take care of the choice of the functions $\left\{F_{k}\right\}$. Let $D$ be a countable dense subset of $C(X)$. We define $\mathscr{G}$ to be the collection of all functions of the form

$$
F(\theta)=f_{1}^{*}(\theta) f_{2}^{*}(\theta) \cdots f_{m}^{*}(\theta),
$$

$m>0$, where $f_{j} \in C(X)$ and as in $\S 5.3$,

$$
f_{j}^{*}(\theta)=\int_{X} f d \theta .
$$

We define $\left\{F_{k}\right\}$ to be the collection of all functions $F_{k} \in \mathscr{G}$ with each $f_{j} \in D$. By the Stone-Weierstrass Theorem, $\mathscr{G}$ generates a dense subspace of $C\left(X^{*}\right)$ and thus $C\left(X^{*} \mid Y\right)$. By (26), $\left\{F_{k}\right\}$ still has this property, and it is also countable.

For $\psi \in C(Y)$ we define a homeomorphism $h^{\psi}$ on $Y \times Z$ by

$$
h^{\psi}(y, z)=\left(y, h^{\psi(y)} z\right),
$$

where $h^{s}$ is the flow from the statement of Theorem 8.4.

Lemma 8.8. If $\varphi=\psi \circ S-\psi$ is a coboundary then $\varphi \in \bar{E}_{F, \varepsilon}$ if and only if $0 \in \bar{E}_{F \circ\left(h^{\psi}\right)^{*}, \varepsilon}$.

Proof. If $\varphi=\varphi^{\prime}+\psi \circ S-\psi$ then

$$
T_{\varphi}^{j}=h^{\psi} \circ T_{\varphi^{\prime}}^{j} \circ h^{-\psi} .
$$

Using (47) with (25), we have

$$
\begin{aligned}
F\left(T_{\varphi}^{* j} \theta\right) & =F\left(\left(h^{\psi}\right)^{*} \circ T_{\varphi^{\prime}}^{* j} \circ\left(h^{-\psi}\right)^{*} \theta\right) \\
& \left.=\left(F \circ\left(h^{\psi}\right)^{*}\right)\left(T_{\varphi^{\prime}}^{* j}\left(h^{-\psi}\right)^{*} \theta\right)\right) .
\end{aligned}
$$


Letting

$$
G_{j}(\theta)=\frac{1}{n+1} \sum_{j=0}^{n} F\left(T_{\varphi}^{* j} \theta\right)-F(\nu \times \omega),
$$

and

$$
G_{j}^{\prime}(\theta)=\frac{1}{n+1} \sum_{j=0}^{n}\left(F \circ\left(h^{\psi}\right)^{*}\right)\left(T_{\varphi^{\prime}}^{* j} \theta\right)-F(\nu \times \omega),
$$

then (48) implies

$$
G_{j}^{\prime}\left(\left(h^{-\psi}\right)^{*} \theta\right)=G_{j}(\theta) .
$$

Since $\left(h^{-\psi}\right)^{*}$ is a homeomorphism of $X^{*} \mid Y$, this implies

$$
\left\|G_{j}\right\|=\left\|G_{j}^{\prime}\right\|,
$$

which implies $\varphi \in E_{F, \varepsilon}$ if and only if $\varphi^{\prime} \in E_{F \circ\left(h^{\psi}\right)^{*}, \varepsilon}$. In other words,

$$
E_{F, \varepsilon}=E_{F \circ \psi, \varepsilon}+(\psi \circ S-\psi) \text {. }
$$

This implies $\varphi_{1} \in \bar{E}_{F, \varepsilon}$ if and only if $\varphi_{1}^{\prime} \in \bar{E}_{F \circ \psi, \varepsilon}$, where $\varphi_{1}=\varphi_{1}^{\prime}+\psi \circ S-\psi$. The lemma follows by taking $\varphi_{1}^{\prime}=0$.

To show $0 \in \bar{E}_{F \circ\left(h^{\psi}\right)^{*}, \varepsilon}$ it is enough to show that for any $\delta>0$ there exists $\varphi \in E_{F \circ\left(h^{\psi}\right)^{*}, \varepsilon}$ with $\|\varphi-0\|<\delta$. Thus the proof of Theorem 8.4 comes down to the following lemma.

Lemma 8.9. Let $k \geq 0$ and $\beta \in C(Y)$. Then for any $\varepsilon>0, \delta>0$ there exists a function $\psi \in C(Y)$ so that for $\varphi(y)=\psi(S y)-\psi(y) \in \mathscr{B}$

(i) $\|\varphi\|<\delta$, and

(ii) $\varphi \in E_{F_{k} \circ\left(h^{\beta}\right)^{*}, \varepsilon}$.

Proof. Let $F_{k}^{\prime}(\theta)=F_{k}\left(\left(h^{\beta}\right)^{*} \theta\right)$. Then by $(25)$

$$
\begin{aligned}
F_{k}^{\prime}(\theta) & =f_{1}^{*}\left(\left(h^{\beta}\right)^{*} \theta\right) f_{2}^{*}\left(\left(h^{\beta}\right)^{*} \theta\right) \cdots f_{m}^{*}\left(\left(h^{\beta}\right)^{*} \theta\right) \\
& =\left(f_{1} \circ h^{\beta}\right)^{*}(\theta)\left(f_{2} \circ h^{\beta}\right)^{*}(\theta) \cdots\left(f_{m} \circ h^{\beta}\right)^{*}(\theta) \\
& =\left(f_{1}^{\prime}\right)^{*}(\theta)\left(f_{2}^{\prime}\right)^{*}(\theta) \cdots\left(f_{m}^{\prime}\right)^{*}(\theta),
\end{aligned}
$$

where $f_{j}^{\prime}(x)=f_{j}\left(h^{\beta} x\right)$. Thus for simplicity we will drop all the primes from now on, denoting $F_{K}^{\prime}$ by $F_{k}$ and $f_{j}^{\prime}$ by $f_{j}$.

To show (ii) for a given $\varphi$, we need to show that there exists $n>0$ such that for all $\theta$,

$$
\left|\frac{1}{n+1} \sum_{j=0}^{n} F_{k}\left(T_{\varphi}^{* j} \theta\right)-F_{k}(\nu \times \omega)\right|<\varepsilon .
$$

Since $\theta \in X^{*} \mid Y$, the product measure $\theta^{m} \in\left(X^{m}\right)^{*} \mid Y^{m}$, and we have the disintegration $\theta=\int_{Y} \theta_{y}(z) d \nu(z)$ and

$$
\theta^{m}=\int_{Y^{m}}\left(\theta^{m}\right)_{\mathbf{y}}(\mathbf{z}) d \nu^{m}(\mathbf{z})
$$


where $\mathbf{y}=\left(y_{1}, \ldots, y_{m}\right)$, and $\mathbf{z}=\left(z_{1}, \ldots, z_{m}\right)$. We have

$$
\begin{aligned}
& \frac{1}{n+1} \sum_{j=0}^{n} F_{k}\left(T_{\varphi}^{* j} \theta\right)=\frac{1}{n+1} \sum_{j=0}^{n} f_{1}^{*}\left(T_{\varphi}^{* j} \theta\right) f_{2}^{*}\left(T_{\varphi}^{* j} \theta\right) \cdots f_{m}^{*}\left(T_{\varphi}^{* j} \theta\right) \\
& =\frac{1}{n+1} \sum_{j=0}^{n} \int_{X} f_{1}\left(T_{\varphi}^{j} x\right) d \theta \int_{X} f_{2}\left(T_{\varphi}^{j} x\right) d \theta \cdots \int_{X} f_{m}\left(T_{\varphi}^{j} x\right) d \theta \\
& \quad=\frac{1}{n+1} \sum_{j=0}^{n} \int_{X^{m}} f_{1}\left(T^{j} x_{1}\right) f_{2}\left(T^{j} x_{2}\right) \cdots f_{m}\left(T^{j} x_{m}\right) d \theta^{m}\left(x_{1}, \ldots, x_{m}\right) \\
& =\frac{1}{n+1} \sum_{j=0}^{n} \int_{Y^{m}} \int_{Z^{m}} f_{1}\left(S^{j} y_{1}, h^{\psi\left(S^{j+1} y_{1}\right)} h^{-\psi\left(y_{1}\right)} z_{1}\right) \\
& \quad \cdots f_{m}\left(S^{j} y_{m}, h^{\psi\left(S^{j+1} y_{m}\right)} h^{-\psi\left(y_{m}\right)} z_{m}\right) d\left(\theta^{m}\right)_{\mathbf{y}}(\mathbf{z}) d \nu^{m}(\mathbf{y}) \\
& =\frac{1}{n+1} \sum_{j=0}^{n} \int_{Y^{m}} \int_{Z^{m}} \phi\left(R^{j} \mathbf{y}, \mathbf{y}, \mathbf{z}\right) d\left(\theta^{m}\right)_{\mathbf{y}}(\mathbf{z}) d \nu^{m}(\mathbf{y}),
\end{aligned}
$$

where

$$
\phi(\mathbf{y}, \mathbf{w}, \mathbf{z})=f_{1}\left(y_{1}, h^{\psi\left(S y_{1}\right)} h^{-\psi\left(w_{1}\right)} z_{1}\right) \cdots f_{m}\left(y_{m}, h^{\psi\left(S y_{m}\right)} h^{-\psi\left(w_{m}\right)} z_{m}\right),
$$

and $R=S \times \cdots \times S$. Notice that $R$ is ergodic since $S$ is weakly mixing. Using (51) with Lemma 8.6 we have

$$
\lim _{n \rightarrow \infty} \frac{1}{n+1} \sum_{j=0}^{n} F_{k}\left(T_{\varphi}^{* j} \theta\right)=\int_{Y^{m} \times Z^{m}} \int_{Y_{m}} \phi(\mathbf{y}, \mathbf{w}, \mathbf{z}) d \nu^{m}(\mathbf{y}) d \theta^{m}(\mathbf{w}, \mathbf{z}) .
$$

Claim. There exists $\varphi \in \mathscr{B}$ satisfying (i) such that for all $\mathbf{w}$ and $\mathbf{z}$,

$$
\left|\int_{Y^{m}} \phi(\mathbf{y}, \mathbf{w}, \mathbf{z}) d \nu^{m}(\mathbf{y})-F_{k}(\nu \times \omega)\right|<\varepsilon / 2 .
$$

To see that the Claim proves the lemma it suffices to show that it implies (ii). Since $\theta^{m}$ is a probability measure (54) implies that the double integral in (53) is within $\varepsilon / 2$ of $F_{k}(\nu \times \omega)$. Thus if we pick $n$ large enough that

$$
\frac{1}{n+1} \sum_{j=0}^{n} F_{k}\left(T_{\varphi}^{* j} \theta\right)
$$

is within $\varepsilon / 2$ of the right-hand side of (53), we have (49), from which (ii) follows.

To prove the Claim, we worry first about (54), leaving (i) for last. Recall that $F_{k}(\theta)=f_{1}^{*}(\theta) \cdots f_{r}^{*}(\theta)$. For any given $\varepsilon_{1}>0$, let $\delta_{1}$ be such that for all $j$,

$$
\left|f_{j}\left(x_{1}\right)-f_{j}\left(x_{2}\right)\right|<\varepsilon_{1},
$$

whenever

$$
d\left(x_{1}, x_{2}\right)<\delta_{1}
$$

We suppose that $\mathscr{P}_{0}=\left\{P_{t} \subseteq Y: t=1, \ldots, N\right\}$ is a finite collection of disjoint subsets of $Y$, each of which is a Cantor set, and such that for all $t$

$$
\operatorname{diam}\left(P_{t}\right)<\delta_{1}
$$


and if $P_{0}=\left(\bigcup_{t=1}^{N} P_{t}\right)^{c}$, then

$$
\nu\left(P_{0}\right)<\varepsilon_{2},
$$

for some given $\varepsilon_{2}$. We will construct $\mathscr{P}_{0}$ below. We let $\mathscr{P}=\mathscr{P}_{0} \cup\left\{P_{0}\right\}$, $\left|\mathscr{P}_{0}\right|=\bigcup_{t=1}^{N} P_{t}$, and for each $t=0, \ldots, N$, we let $p_{t} \in P_{t}$. We define

$$
Q_{t}=Q_{\left(t_{1}, \ldots, t_{r}\right)}=P_{t_{1}} \times \cdots \times P_{t_{m}} .
$$

We put $\mathscr{Q}=\left\{Q_{t}\right\}, \mathscr{Q}_{0}=\left\{Q_{t}: t_{j} \neq 0\right\}$ and $\left|\mathscr{Q}_{0}\right|=\bigcup_{Q_{t} \in \mathscr{Q}_{0}} Q_{t}$. We also put $q_{t}=\left(p_{t_{1}}, p_{t_{2}}, \ldots, p_{t_{m}}\right) \in Q_{t}$.

Let us define $\tilde{f}_{j}(y, z)=f_{j}\left(p_{t}, z\right)$ for $y \in P_{t}, w \in Y$, and $z \in Z$. Since each $\tilde{f}_{j}(y, z)$ is piecewise constant in $y$,

$$
\begin{aligned}
& \int_{Y} \tilde{f}_{j}\left(y, h^{\psi(S y)} h^{-\psi(w)} z\right) d \nu(y) \\
& \quad=\sum_{t} \nu\left(P_{t}\right) \int_{P_{t}} \tilde{f}_{j}\left(p_{t}, h^{\psi(S y)} h^{-\psi(w)} z\right) d \nu(y) .
\end{aligned}
$$

Now it follows from (55), (56), and (57) that

$$
\left|\tilde{f}_{j}\left(y, h^{\psi(S y)} h^{-\psi(w)} z\right)-f_{j}\left(y, h^{\psi(S y)} h^{-\psi(w)} z\right)\right|<\varepsilon_{1},
$$

for all $w \in Y, z \in Z$, and $y \in\left|\mathscr{P}_{0}\right|$. This implies that if we define $\tilde{\phi}$ like $\phi$ in (52), but with each $f_{j}$ replaced by $\tilde{f}_{j}$, then we have for all $\mathbf{w}, \mathbf{z}$, and $\mathbf{y} \in\left|\mathscr{Q}_{0}\right|$,

$$
|\phi(\mathbf{y}, \mathbf{w}, \mathbf{z})-\tilde{\phi}(\mathbf{y}, \mathbf{w}, \mathbf{z})|<C_{1} \varepsilon_{2}<\varepsilon / 12,
$$

provided we have chosen

$$
\varepsilon_{1}<\varepsilon / 12 C_{1} \text {. }
$$

The constant $C_{1}$ depends only on the norms of the functions $f_{j}$, and comes from the proof that multiplication is continuous. It follows from (58) and (59) that $\nu^{m}\left(\left|\mathscr{Q}_{0}\right|\right)>1-m \varepsilon_{2}$. This together with (61) implies

$$
\begin{aligned}
& \left|\int_{Y^{m}} \phi(\mathbf{y}, \mathbf{w}, \mathbf{z}) d \nu^{m}(\mathbf{y})-\int_{Y^{m}} \tilde{\phi}(\mathbf{y}, \mathbf{w}, \mathbf{z}) d \nu^{m}(\mathbf{y})\right| \\
& <\varepsilon_{1}+m\|\phi\| \varepsilon_{2}<\varepsilon / 12+\varepsilon / 12=\varepsilon / 6,
\end{aligned}
$$

using (62), and provided $\varepsilon_{2}$ has been chosen to satisfy

$$
\varepsilon_{2}<\frac{\varepsilon}{12 m\|\phi\|} .
$$

Now let $f_{j}^{t}(z)=\tilde{f}_{j}\left(p_{t}, z\right)=f_{j}\left(p_{t}, z\right)$. By the unique ergodicity of $h^{s}$, for any $\varepsilon_{3}>0$ there exists a positive real number $L$ large enough that

$$
\left|\frac{1}{L} \int_{0}^{L} f_{j}^{t}\left(h^{s} z\right) d s-\int_{Z} f_{j}^{t}(z) d \omega(z)\right|<\varepsilon_{3},
$$

uniformly in $j$ and $t$.

Let us suppose that for each Cantor set $P_{t} \in \mathscr{P}_{0}$, we have a continuous function $\tau_{t}$ on $S\left(P_{t}\right)$ which satisfies (42) from Lemma 8.7, where $L$ is given by (65). We define $\tau$ on $\left|\mathscr{P}_{0}\right|$ to be $\tau_{t}$ on each set $P_{t} \in \mathscr{P}_{0}$, and suppose 
that $\tau: Y \rightarrow[0, L]$ is an arbitrary continuous extension of $\left.\tau\right|_{\mathscr{P}_{0}}$. We define $\psi=\tau \circ S^{-1}$ so that $\psi \circ S=\tau$. Using a change of variables

$$
\begin{aligned}
\int_{P_{t}} \tilde{f}_{j}\left(y, h^{\psi(S y)} h^{-\psi(w)} z\right) d \nu(y) & =\int_{P_{t}} f_{j}^{t}\left(h^{\psi(S y)} h^{-\psi(w)} z\right) d \nu(y) \\
& =\int_{P_{t}} f_{j}^{t}\left(h^{\tau_{t}(y)}\left(h^{-\psi(w)} z\right)\right) d \nu(y) \\
& =\int_{0}^{L} f_{j}^{t}\left(h^{s}\left(h^{-\psi(w)} z\right)\right) \frac{d s}{L},
\end{aligned}
$$

so that by (65) and (66) we have

$$
\left|\int_{P_{t}} \tilde{f}_{j}\left(y, h^{\psi(S y)} h^{-\psi(w)} z\right) d \nu(y)-\int_{Z} f_{j}^{t}\left(z_{1}\right) d \omega\left(z_{1}\right)\right|<\varepsilon_{3},
$$

for all $j, w, v$, and $t \neq 0$. Using the fact that $\tilde{f}_{j}(y, z)$ is piecewise constant in $y$, and summing (67), we have

$$
\begin{aligned}
\sum_{t} \nu\left(P_{t}\right) \int_{Z} f_{j}^{t}(z) d \omega(z) & =\int_{Z} f_{j}^{t}(z) d \omega(z) \\
=\int_{Z} \tilde{f}_{j}\left(p_{t}, z\right) d \omega(z) & =\int_{Y \times Z} \tilde{f}_{j}(y, z) d(\nu \times \omega) .
\end{aligned}
$$

Combining (60), (67), and (68) yields

$$
\left|\int_{Y} \tilde{f}_{j}\left(y, h^{\psi(S y)} h^{-\psi(w)} z\right) d \nu(y)-\int_{Y \times Z} \tilde{f}_{j}(y, z) d(\nu \times \omega)(y, z)\right|<\varepsilon_{3},
$$

for all $j$. It follows from (69) that

$$
\begin{aligned}
& \left|\int_{Y^{m}} \tilde{\phi}(\mathbf{y}, \mathbf{w}, \mathbf{z}) d \nu^{m}(\mathbf{y})-\int_{X^{m}} \tilde{f}_{1}\left(y_{1}, z_{1}\right) \cdots \tilde{f}_{m}\left(y_{m}, z_{m}\right) d(\nu \times \omega)^{m}(\mathbf{y}, \mathbf{z})\right| \\
& \quad<C_{1} \varepsilon_{3}<\varepsilon / 6
\end{aligned}
$$

provided $\varepsilon_{3}$ has been chosen so that

$$
\varepsilon_{2}<\varepsilon / 6 C_{1},
$$

where $C_{1}$ is as in (61).

Now we concentrate on the right-hand side of (70). For $y \in P_{t}, t \neq 0$, we have by (57),

$$
d\left(\left(p_{t}, z\right),(y, z)\right)=d\left(p_{t}, y\right)<\delta_{1},
$$

and it follows from (55) that

$$
\left|\tilde{f}_{j}(y, z)-f_{j}(y, z)\right|<\varepsilon_{1},
$$

for $(y, z) \in\left|\mathscr{P}_{0}\right| \times Z$. This implies

$$
\begin{aligned}
& \left|\int_{Z} \tilde{f}_{j}(y, z) d \omega(z)-\int_{Z} f_{j}(y, z) d \omega(z)\right| \\
& \quad \leq \int_{Z}\left|\tilde{f}_{j}(y, z)-f_{j}(y, z)\right| d \omega(z)<\varepsilon_{1},
\end{aligned}
$$


for all $y \in\left|\mathscr{P}_{0}\right|$, which when combined with (58), implies

$$
\begin{aligned}
& \left|\int_{Y \times Z} \tilde{f}_{j}(y, z) d(\nu \times \omega)(y, z)-\int_{Y \times Z} f_{j}(y, z) d(\nu \times \omega)(y, z)\right| \\
& \quad<\varepsilon_{1}+\left\|f_{j}\right\| \varepsilon_{2}<\varepsilon /\left(6 C_{1}\right),
\end{aligned}
$$

by choosing $\varepsilon_{2}$ to satisfy

$$
\varepsilon_{2}<\varepsilon / 6 M C_{1},
$$

in addition to (62). Here $M$ is the maximum of $\left\|f_{j}\right\|$. Then (72) implies

$$
\begin{gathered}
\mid \int_{X^{m}} \tilde{f}_{1}\left(y_{1}, z_{1}\right) \cdots \tilde{f}_{m}\left(y_{m}, z_{m}\right) d(\nu \times \omega)^{m}(\mathbf{y}, \mathbf{z}) \\
-\int_{X^{m}} f_{1}\left(y_{1}, z_{1}\right) \cdots f_{m}\left(y_{m}, z_{m}\right) d(\nu \times \omega)^{m}(\mathbf{y}, \mathbf{z}) \mid \\
<C_{1} \cdot\left(\frac{\varepsilon}{6 C_{1}}\right)=\varepsilon / 6 .
\end{gathered}
$$

We note that by (45) and (46), the right-hand side of (74) is equal to $F_{k}(\nu \times \omega)$. Thus, a combination of (61), (70), and (75) yields equation (54) of the Claim.

It remains to construct $\mathscr{P}_{0}$ to satisfy equations (57) and (58), and $\tau_{t}$ to satisfy (42) on each $P_{t}$, so that $\varphi=\psi \circ S-\psi=\tau-\tau \circ S^{-1}$ satisfies (i). Choose a positive integer

$$
K>\max \left\{\frac{2}{\varepsilon}, \frac{2 L}{\delta}\right\}
$$

where $\delta$ comes from (i), and $L$ comes from (65). By the Rohlin Lemma there exist disjoint Cantor sets $A_{1}, A_{2}, \ldots, A_{K^{2}}$ with $A_{j}=S^{j-1} A_{1}$, such that $\nu\left(\bigcup_{j=1}^{K^{2}} A_{j}\right)>1-\varepsilon_{2} / 2$. We partition $A_{1}=\bigcup_{i=1}^{J} A_{1, i}$ so that for $j=1, \ldots, K^{2}$, $i=1, \ldots, J$, if $A_{j, i}=S^{j-1} A_{1, i}$ then $\operatorname{diam}\left(A_{j, i}\right)<\delta_{1}$. We define $\mathscr{P}_{0}^{\prime}=\left\{P_{t}^{\prime}\right.$ : $t>0\}=\left\{A_{j, k}: 1 \leq j \leq K^{2}, 1 \leq i \leq J\right\}$. For $P_{t}^{\prime}=A_{1, i}$, we apply Lemma 8.7 to define functions $\tau_{t}^{\prime}$ satisfying (42) on each $P_{t}^{\prime}$. We can use Lemma 8.7 because $S$ is weakly mixing, and this implies that $\nu$ is nonatomic.

For $P_{t^{\prime}}^{\prime}=A_{j, i}=S^{j-1} A_{1, i}=S^{j-1} P_{t}^{\prime}$, where $j>1$, we define

$$
\tau_{t^{\prime}}^{\prime}=\tau_{t}^{\prime} \circ S^{-j+1} \text {. }
$$

This defines $\tau^{\prime}$ on $\mathscr{P}_{0}^{\prime}$ in such a way that (42) is still satisfied, and we extend $\tau^{\prime}$ in an arbitrary way to a $[0, L]$-valued continuous function on $Y$.

Finally we let

$$
\tau(y)=\frac{1}{K} \sum_{j=0}^{K-1} \tau^{\prime}\left(S^{j} y\right),
$$

so that by (78) and (77), $\tau=\tau^{\prime}$ on all the levels of the Rohlin tower, except the last $K$ levels. In particular (42) holds on the first $K^{2}-K$ levels. Thus if we let $\mathscr{P}_{0}$ consist of the sets $P_{t}^{\prime}$ in $\mathscr{P}_{0}^{\prime}$ which are subsets of the first $K^{2}-K$ levels, then (42) holds on $\mathscr{P}_{0}$. It follows from (76) that (58) is satisfied. Finally we have by (78)

$$
\begin{aligned}
\|\varphi\| & =\|\psi \circ S-\psi\|=\left\|\tau-\tau \circ S^{-1}\right\| \\
& =\frac{1}{K}\left\|\tau^{\prime} \circ S^{K-1}-\tau^{\prime} \circ S^{-1}\right\| \leq \frac{2 L}{K}<\delta,
\end{aligned}
$$

where (79) follows from (76). 


\section{Discussion}

As we noted above, we have been unable to determine whether or not stable extensions lift the Bernoulli property. This seems to come down to two questions. The first is to determine the degree to which the Bernoulli property can be characterized in terms of joinings, a question already answered to a large extent by Rothstein and Burton, [4]. The second question is whether stability is a strong enough lifting condition for this purpose.

Clearly stability is not the strongest lifting condition satisfied by group extensions. For example, it is not sufficient to lift quasi-disjointness, a property studied by Berg, [1, 2], which does lift to group extensions. (Quasi-disjointness essentially amounts to disjointness modulo the maximal common discrete spectrum factor.) While it is easy to see how to strengthen the definition of stability to deal with this case, the Bernoulli case is less clear. Moreover, there is good reason to worry that a substantially different approach will be needed to handle the Bernoulli case. Using techniques from [33], assuming only very mild conditions on the cocycle, Rudolph [34] constructed examples of smooth flow extensions to obtain $K$-transformations which are not even loosely Bernoulli.

A different area where it is unclear whether or not stability is sufficient lifting condition is in the theory of ergodic multiplier properties (cf. [29]). Although these properties are closely related to rigidity via [14], the lifting theorem for ergodic multiplier properties is not known even in the case of nonabelian group extensions. A related question is whether these ergodic multiplier properties are natural properties.

Finally, since one of the main motivations for studying extensions is to be able to construct examples, it would be useful to have some smooth examples of stable extensions other than group extensions. This is probably possible, although we have not yet tried it.

\section{REFERENCES}

1. K. Berg, Quasidisjointness in ergodic theory, Trans. Amer. Math. Soc. 162 (1971), 71-87.

2. $\ldots$, Quasidisjointness, products and inverse limits, Math. Systems Theory 6 (1972), 123128.

3. J. R. Blum and D. L. Hanson, On the main ergodic theorem for subsequences, Bull. Amer. Math. Soc. 66 (1963), 308-311.

4. A. Rothstein and R. Burton, Isomorphism theorems in ergodic theory, Lecture notes, Department of Mathematics, Oregon State University.

5. J. Coquet and P. Lairdet, A metric study involving independent sequences, J. Analyse Math. 49 (1987), 15-53.

6. N. Friedman, Mixing on sequences, Canad. J. Math. 35 (1983), 339-352.

7. __, Higher order partial mixing, Contemp. Math., vol. 26, Amer. Math. Soc., Providence, R.I., 1984, pp. 111-130.

8. N. Friedman and D. Ornstein, On partial mixing transformations, Indiana Univ. Math. J. 20 (1971), 767-775.

9. __ On mixing and partial mixing, Illinois J. Math. 16 (1972), 61-68.

10. H. Furstenberg, Disjointness in ergodic theory, minimal sets, and a problem in Diophantine approximation, Math. Systems Theory 1 (1967), 1-49.

11. __ Strict ergodicity and transformations of the torus, Amer. J. Math. 83 (1961), 573-601.

12. __ Ergodic behavior of diagonal measures and a theorem of Szemerédi on arithmetic progressions, J. Analyse Math. 31 (1977), 204-256. 
13. __ Recurrence in ergodic theory and combinatorial number theory, Princeton Univeristy Press, Princeton, N.J., 1981.

14. H. Furstenberg and B. Weiss, The finite multipliers of infinite ergodic transformations, Lecture Notes in Math., vol. 668, Springer-Verlag, Berlin and New York, 1978, pp. 127-132.

15. S. Glasner, Relatively invariant measures, Pacific. J. Math. 58 (1975), 393-410.

16. __ Quasi-factors in ergodic theory, Israel J. Math. 45 (1983), 198-208.

17. S. Galsner and B. Weiss, On the construction of minimal skew products, Israel J. Math. 34 (1979), 321-336.

18. __ Processes disjoint from weak mixing, Trans. Amer. Math. Soc. 316 (1989), 689-703.

19. K. L. Jones, A mean ergodic theorem for weakly mixing sequences of operators, Adv. in Math. 7 (1971), 211-216.

20. R. Jones and W. Parry, Compact abelian group extensions of discrete dynamical systems II, Comput. Math. 25 (1972), 135-147.

21. J. King, Joining-rank and the structure, J. Analyse Math. 51 (1988), 182-221.

22. W. Krieger, On unique ergodicity, Proc. Sixth Berkeley Sympos., Univ. of California Press, 1972, pp. 327-346.

23. G. W. Mackey, Borel structures in groups and their duals, Trans. Amer. Math. Soc. 85 (1957), 134-165.

24. E. E. Moise, Geometric topology in dimensions 2 and 3, Springer-Verlag, New York and Berlin, 1977.

25. M. Nerurkar, Generic theorems for continuous, ergodic skew product actions of amenable groups, Pacific J. Math. 119 (1985), 343-363.

26. W. Parry, Ergodic properties of transformations and flows on nilmanifolds, Amer. J. Math. 91 (1969), 757-771.

27. M. S. Pinsker, Dynamical systems with completely positive or zero entropy, Dokl. Akad. Nauk SSSR 133 (1960), 1025-1026; English transl. in Soviet Math. Dokl. 1 (1961), 335-338.

28. E. A. Robinson, The maximal abelian subextension determines weak mixing for group extensions, Proc. Amer. Math. Soc. (to appear).

29. Ergodic properties that lift to compact group extensions, Proc. Amer. Math. Soc. 102 (1988), 61-67.

30. D. Rudolph, An example of a measure preserving transformation with minimal self-joinings, J. Analyse Math. 35 (1979), 97-122.

31. __ Classifying the isometric extensions of a Bernoulli shift, J. Analyse Math. 34 (1978), 36-60.

32. _,$K$-fold mixing lifts to weakly mixing isometric extensions, Ergodic Theory Dynamical Systems 5 (1985), 445-447.

33. —, $\mathbf{Z}^{n}$ and $\mathbf{R}^{n}$ cocycle extensions and complementary algebras, Ergodic Theory Dynamical Systems 6 (1986), 583-599.

34. __ Asymptotically Brownian skew products give nonloosely Bernoulli K-automorphisms, preprint.

35. J.-P. Thouvenot, Quelques propriétés des systèms dynamiques qui se décomposant en un produit de deux systèmes dont l un es un schèma de Bernoulli, Israel J. Math. 21 (1975), 177-207.

36. H. Totoki, Ergodic theory, Aarhus Univ. Lecture Notes, 1969.

37. V. S. Varadarajan, Groups of automorphisms of Borel spaces, Trans. Amer. Math. Soc. 109 (1963), 191-220.

38. P. Walters, An introduction to ergodic theory, Springer-Verlag, New York and Berlin, 1982.

39. _ Some invariant $\sigma$-algebras for measure-preserving transformations, Trans. Amer. Math. Soc. 163 (1972), 357-368.

40. __ Some transformations having a unique measure with maximal entropy, Proc. London Math. Soc. (3) 28 (1974), 500-516. 
41. B. Weiss, Strictly ergodic models for dynamical systems, Bull. Amer. Math. Soc. 13 (1985), 143-146.

42. R. Zimmer, Extensions of ergodic group actions, Illinois J. Math. 20 (1976), 373-409.

43. $\ldots$, Ergodic actions with generalized discrete spectrum, Illinois J. Math. 20, (1976), 555588.

Department of Mathematics, The George Washington University, Washington, D.C. 20052 